\title{
The Dependence of Bar Frequency on Galaxy Mass, Colour, and Gas Content - and Angular Resolution - in the Local Universe
}

\author{
Peter Erwin ${ }^{1,2 \star}$ \\ ${ }^{1}$ Max-Planck-Insitut für extraterrestrische Physik, Giessenbachstrasse, 85748 Garching, Germany \\ ${ }^{2}$ Universitäts-Sternwarte München, Scheinerstrasse 1, D-81679 München, Germany
}

Accepted XXX. Received YYY; in original form ZZZ

\begin{abstract}
I use distance- and mass-limited subsamples of the Spitzer Survey of Stellar Structure in Galaxies $\left(\mathrm{S}^{4} \mathrm{G}\right)$ to investigate how the presence of bars in spiral galaxies depends on mass, colour, and gas content and whether large, SDSS-based investigations of bar frequencies agree with local data. Bar frequency reaches a maximum of $f_{\mathrm{bar}} \approx 0.70$ at $M_{\star} \sim 10^{9.7} M_{\odot}$, declining to both lower and higher masses. It is roughly constant over a wide range of colours $(g-r \approx 0.1-0.8)$ and atomic gas fractions $\left(\log \left(M_{\mathrm{HI}} / M_{\star}\right) \approx-2.5\right.$ to 1). Bars are thus as common in blue, gas-rich galaxies are they are in red, gaspoor galaxies. This is in sharp contrast to many SDSS-based studies of $z \sim 0.01-0.1$ galaxies, which report $f_{\mathrm{bar}}$ increasing strongly to higher masses (from $M_{\star} \sim 10^{10}$ to $\left.10^{11} M_{\odot}\right)$, redder colours, and lower gas fractions. The contradiction can be explained if SDSS-based studies preferentially miss bars in, and underestimate the bar fraction for, lower-mass (bluer, gas-rich) galaxies due to poor spatial resolution and the correlation between bar size and stellar mass. Simulations of SDSS-style observations using the $\mathrm{S}^{4} \mathrm{G}$ galaxies as a parent sample, and assuming that bars below a threshold angular size of twice the PSF FWHM cannot be identified, successfully reproduce typical SDSS $f_{\text {bar }}$ trends for stellar mass and gas mass ratio. Similar considerations may affect high-redshift studies, especially if bars grow in length over cosmic time; simulations suggest that high-redshift bar fractions may thus be systematically underestimated.
\end{abstract}

Key words: galaxies: structure - galaxies: elliptical and lenticular, $c \mathrm{D}$ - galaxies: bulges - galaxies: spiral

\section{INTRODUCTION}

Stellar bars are an important - and often visually striking - component of many disc galaxies. They are believed to be key drivers of secular evolution, in part because they can act as both sources and sinks for angular momentum, redistributing stars, gas, and dark matter within galaxies ( e.g., Athanassoula 2002, 2003; Weinberg \& Katz 2002; Kormendy \& Kennicutt 2004; Holley-Bockelmann et al. 2005; Debattista et al. 2006; Sellwood 2014). Perhaps the most visible aspect of this is bar-driven gas inflow and subsequent star formation in the central regions of galaxies, which has been suggested to play a key role in building up bulges and pseudobulges. Bar-driven gas inflow has also long been implicated in the fuelling of AGN (although confirmation of this last idea has proved somewhat elusive; e.g., Shlosman

* E-mail: erwin@mpe.mpg.de et al. 1989, 1990; Ho et al. 1997; Laine et al. 2002; Lee et al. 2012b; Cheung et al. 2015; Goulding et al. 2017).

In addition to their direct effects on their host galaxies, the mere presence or absence of bars can provide important insights into galactic evolution. The discovery from early simulations that $N$-body discs routinely become barunstable raised the question of why some disc galaxies did not have bars. The demonstration that massive halos could stabilize discs against bar formation (e.g., Ostriker \& Peebles 1973; Hohl 1976) was one reason for the growing acceptance of dark-matter halos in the 1970s, and bar-instability criteria, especially based on Efstathiou et al. (1982), have played a major role in semi-analytic models of galaxy evolution (e.g., Mo et al. 1998). Ironically, subsequent numerical experiments with live dark-matter halos have shown that halos can actually amplify bar growth, since halo particles can absorb angular momentum from bar instabilities and help them grow (e.g., Athanassoula 2002; Athanassoula 
2008; Saha et al. 2013; Sellwood 2016). Nevertheless, the fact that instabilities in sufficiently cool discs readily give rise to bars means that the appearance of bars in galaxies at high redshifts can be a useful - and easily visible - indicator of when and how rapidly galaxy discs become kinematically cool (e.g., Sheth et al. 2012).

Bars are thus important both for how they can reshape galaxies and as general diagnostics for the evolution of discs. But in order to have a clear understanding of how important bars really are for galaxy evolution in general, we need to know what fraction of discs really do (or do not) have bars. Ideally, we also want to know what kinds of discs are more (or less) likely to host bars.

In the local universe (roughly, closer than $\sim 50 \mathrm{Mpc}$ ), the fraction of disc galaxies with stellar bars has been reported to be roughly $2 / 3$ (or $1 / 3$ if only so-called "strong" bars are counted). A nice overview of past studies can be found in the Introduction of Sheth et al. (2008); they write, "We conclude that the consensus value of the local barred fraction is $f_{\text {bar }} \sim 0.3$ for strongly barred systems, and $f_{\text {bar }} \sim 0.65$ for all barred galaxies, and that these values are so well known that they have not changed significantly in over four decades."

The advent of multi-band imaging (and accompanying spectroscopy) from the Sloan Digital Sky Survey (SDSS; York et al. 2000) has opened up impressively large new fields of analysis for the moderately nearby universe (i.e., beyond $\sim 50 \mathrm{Mpc}$ and out to $z \sim 0.1$ ). Studies using increasingly large numbers of galaxies have looked at the question of how common bars are, beginning with Barazza et al. (2008) and Aguerri et al. (2009), who analyzed samples of 1144 and 3060 low-inclination discs, respectively, and then Nair \& Abraham (2010a,b), using approximately 7700 galaxies (out of a larger sample of 14,700 galaxies). The largest samples have come from successors to the Galaxy Zoo project (Lintott et al. 2008), in particular Galaxy Zoo 2 (hereafter GZ2 Willett et al. 2013), with samples of between 13,000 and 15,000 low-inclination discs and bar classifications via an innovative citizen-science approach (e.g., Masters et al. 2011; Skibba et al. 2012; Cheung et al. 2013). These studies have generally found rather low bar fractions: most often $\sim 25-30 \%$, though values as high as $\sim 50 \%$ have come from some surveys (e.g., Barazza et al. 2008; Aguerri et al. 2009; Yoshino \& Yamauchi 2015). Several of the SDSS-based studies have cautioned that their results are perhaps applicable primarily to "strong" bars, however those might be defined. The fact that the local strong-bar (SB) frequency is $\sim 30 \%$ makes this an appealing argument, since it more or less matches the most common SDSS-based bar frequencies.

Many of these studies have also investigated what kinds of disc galaxies are more - or less - likely to host bars. This has yielded some rather striking results: bars are apparently much more common in red, massive, and gas-poor galaxies than they are in blue, low-mass, and gas-rich galaxies (e.g., Masters et al. 2011, 2012; Skibba et al. 2012; Oh et al. 2012; Lee et al. 2012a; Cheung et al. 2013; Gavazzi et al. 2015; Consolandi 2016; Cervantes Sodi 2017). Only a few SDSS-based studies have found different - or more complex - trends (e.g., Barazza et al. 2008; Nair \& Abraham 2010b; Méndez-Abreu et al. 2012).

Studies using HST imaging (e.g., Abraham et al. 1999; Sheth et al. 2003; Jogee et al. 2004; Elmegreen et al. 2004;
Sheth et al. 2008; Cameron et al. 2010; Sheth et al. 2012; Melvin et al. 2014; Simmons et al. 2014) have looked at how common bars are at higher redshifts (mostly $z \sim 0.4-1.0$ ), to see if we can observe evolution in the bar fraction. Various methodologies for bar detection have been used, including a version of the GZ2 citizen-science approach (Melvin et al. 2014; Simmons et al. 2014). Most of these studies have reported a clear decrease in the bar fraction toward higher redshifts. At least some have found evidence that at any given redshift there are more bars in higher-mass galaxies than in lower-mass galaxies, and also that the bar fraction reaches present-day levels first for higher-mass samples (Sheth et al. 2008; Melvin et al. 2014). This seems consistent with the strong mass-dependence of bar frequency seen in the SDSS studies, as if the bar fraction saturated for high-mass galaxies some time ago, and is still low (and increasing?) for lowermass galaxies.

The Spitzer Survey of Stellar Structure in Galaxies $\left(S^{4} \mathrm{G}\right.$; Sheth et al. 2010) has made available high-S/N nearIR images for over 2000 local galaxies. Approximately 1300 of these are disc galaxies with inclinations low enough for reliable detection and analysis of bars. Díaz-García et al. (2016a,b) have recently presented a detailed analysis of bars identified by Buta et al. (2015) and measured by HerreraEndoqui et al. (2015). Overall, the local bar fraction is apparently still in the $60-70 \%$ range; Buta et al. (2015) report a bar fraction of "about 2/3". The upper panel of Fig. 19 in Díaz-García et al. (2016a) shows how bar frequency in the entire face-on sample depends on stellar mass: there is a general increase in $f_{\text {bar }}$ from the lowest stellar masses to a broad peak or plateau with $f_{\text {bar }} \sim 0.6$, extending from $\log \left(M_{\star} / M_{\odot}\right) \sim 9.2-10.6$, with a weak maximum at $\sim 9.7$. This disagrees with most of the SDSS-based studies. In particular, the strong increase in $f_{\text {bar }}$ from $\log \left(M_{\star} / M_{\odot}\right) \sim 10$ to 11 seen for most large SDSS samples (Nair \& Abraham 2010b; Masters et al. 2012; Oh et al. 2012; Skibba et al. 2012; Melvin et al. 2014) is not seen in the local sample, nor is the strong increase in $f_{\mathrm{bar}}$ to lower stellar masses reported by Nair \& Abraham (2010b).

We have, therefore, several related puzzles. Can we resolve the apparent dichotomy in overall bar frequency between local samples $\left(f_{\text {bar }} \sim 0.65\right)$ and larger, more distant SDSS-based samples $\left(f_{\text {bar }} \sim 0.25-0.5\right)$ ? Is the difference just due to the the SDSS studies only detecting strong bars? Do the apparently strong correlations between bar frequency and stellar mass, colours, and gas richness seen in the SDSS studies also exist for local galaxies? If not, why not? And what, if anything, does this imply for studies of bars at high redshifts?

In this paper, I investigate these questions by seeing whether $\mathrm{S}^{4} \mathrm{G}$ galaxies - restricted to volume- and masslimited subsamples and with added data from the literature (Section 2) - show the same bar-frequency trends with stellar mass, colour, and gas richness that the large SDSS samples do. Since the answer to this question turns out to be a rather strong "No" (Section 3), I focus on whether the disagreement could be due to problems of spatial resolution, given that the average galaxy in an SDSS-based survey is almost ten times further away than the average $S^{4} \mathrm{G}$ galaxy. This requires taking a closer look at bar sizes, which prove (as previously noted by Díaz-García et al. 2016a) to have a strong dependence on stellar mass (Section 4). I then argue that a depen- 
dence of bar size on stellar mass can straightforwardly translate into a mass-dependent, differential bar-detection bias, and that the combination of the actual sizes of bars and the typical resolution of SDSS images make this potential bias a very real possibility, one which could explain the typical dependence of bar frequency on stellar mass seen in SDSSbased studies. Since colour and gas richness (and certain other properties such as specific star-formation rate) have strong correlations with stellar mass, this can also explain, at least partly, the reported dependence of bar frequency on colour, gas mass ratio, and star-formation rates. By treating the $\mathrm{S}^{4} \mathrm{G}$ galaxies as parent sample and generating bootstrapped mock samples of galaxies observed at typical SDSS distances, I show (Section 5) that the reported SDSS-based bar-fraction trends for stellar mass and gas mass ratio can be qualitatively - and least partly quantitatively - generated just by assuming that bars with projected angular sizes less than twice the FWHM of the PSF go undetected. I consider some additional issues - in particular, the implications for high-redshift studies of bar frequencies - in Section 6 .

Where necessary, I assume a standard $\Lambda$ CDM cosmology based on Bennett et al. (2013), with $H_{0}=70 \mathrm{~km} \mathrm{~s}^{-1}$ $\mathrm{Mpc}^{-1}, \Omega_{m}=0.29$, and $\Omega_{\Lambda}=0.71$.

To aid in reproducibility, there is a Github repository containing data files, code, and Jupyter notebooks for creating the figures, fits, and simulations used in this paper. The repository is available at https://github.com/perwin/ s4g_barfractions and also at https://doi.org/10.5281/ zenodo. 804909 .

\section{DATA AND SAMPLE DEFINITIONS FOR LOCAL GALAXIES}

\subsection{Parent Sample and Data Sources}

The best local sample for assessing bar frequencies and correlations is undoubtedly the Spitzer Survey of Stellar Structure in Galaxies ( $\mathrm{S}^{4} \mathrm{G}$; Sheth et al. 2010), both because of its size and because of its use of near-infrared imaging, which minimizes the possibility of missing bars due to the confusion introduced by dust extinction and star formation. The entire sample has been subjected to a consistent classical morphological analysis - including bar classifications - by Buta et al. (2015), with extensive quantitative bar measurements and analyses by Herrera-Endoqui et al. (2015) and Díaz-García et al. (2016a,b). It does suffer from being a magnitude- and diameter-limited sample (e.g., Figure 1), which prevents it from being fully volume-limited; however, the relatively faint magnitude limit $(B \leqslant 15.5)$ ensures that reasonable volumeand mass-limited subsamples can still be derived from it; the details of this are discussed in Section 2.2.

I start with the $\mathrm{S}^{4} \mathrm{G}$ subsample of non-edge-on disc galaxies defined by Díaz-García et al. (2016a). This takes the version of $\mathrm{S}^{4} \mathrm{G}$ classified by Buta et al. (2015) and then eliminates elliptical galaxies and disc galaxies with inclinations $>65^{\circ}$, leaving a total of 1344 galaxies. Distances and stellar masses for these galaxies are taken from Muñoz-Mateos et al. (2015). The distances are based on redshift-independent NED distances for $79 \%$ of the total sample, and on Hubbleflow distances for the rest (assuming $H_{0}=71$ ). Ten galaxies missing distances and stellar masses in Muñoz-Mateos et al.
(2015) were then removed, along with twelve more which had very uncertain distances (i.e., redshifts $<500 \mathrm{~km} \mathrm{~s}^{-1}$ and no alternate distance measurements) or optical diameters smaller than the original $\mathrm{S}^{4} \mathrm{G}$ limit $\left(D_{25}=1.0^{\prime}\right)$, leaving a total of 1322 galaxies. This is the Parent Disc Sample.

The $\mathrm{S}^{4} \mathrm{G}$ sample as studied in the literature suffers from incompleteness in terms of Hubble types, stemming from the fact that it was defined to have a redshift limit of 3000 $\mathrm{km} \mathrm{s}^{-1}$ using radio radial velocities. Galaxies without such velocities were thus excluded, even if they had optical radial velocities that would put them in the sample. This limitation creates a morphological bias: gas-poor early-type galaxies, including both ellipticals and S0s, are much more likely to be missing than are spiral galaxies. ${ }^{1}$ Using the February 2017 version of HyperLeda and the basic $S^{4} \mathrm{G}$ sample definition of Sheth et al. (2010), there are 167 S0 galaxies with $V_{\text {radio }}<3000 \mathrm{~km} \mathrm{~s}^{-1}$ (156 of which are in the complete $\mathrm{S}^{4} \mathrm{G}$ sample ${ }^{2}$ ) and 315 without $V_{\text {radio }}$ values but with $V_{\text {optical }}<3000 \mathrm{~km} \mathrm{~s}^{-1}$; this contrasts with 2350 spirals and irregulars with $V_{\text {radio }}<3000 \mathrm{~km} \mathrm{~s}^{-1}$ and only 273 without $V_{\text {radio }}$ values. So there is an incompleteness of $\approx 67 \%$ for S0 galaxies versus only $10 \%$ for later types. (Further Spitzer observations to fill in the missing ellipticals and S0s have been obtained by the $\mathrm{S}^{4} \mathrm{G}$ team - e.g., Sheth et al. 2013, Knapen et al. 2014 - but the corresponding morphological analyses and measurements are not yet available, so I confine myself to the original, spiral-dominated sample.)

Since there is some evidence for differences in bars in S0s versus bars in spirals, both in terms of frequencies and also in terms of bar strengths (e.g., Aguerri et al. 2009; Buta et al. 2010; Díaz-García et al. 2016a), it makes sense to separate the highly incomplete S0 subsample from the mostly complete spirals. Consequently, I exclude S0 galaxies from the main samples for analysis in this paper, by retaining only galaxies with optical Hubble type $T>-0.5$. The result is the Parent Spiral Sample, with 1220 galaxies (note that this name is slightly misleading, since the sample includes $\sim 150$ irregular galaxies).

Whether a galaxy is considered barred or not comes from Table 2 of Herrera-Endoqui et al. (2015): if a bar and its accompanying measurements are among the features listed for that galaxy, then it is deemed to be barred. This is in turn based on the visual bar classifications of Buta et al. (2015), except that 117 of the latter galaxies with barred classifications do not have barred measurements in Herrera-Endoqui et al. 2015; these are primarily galaxies with the very weakest Buta et al. 2015 subclassification (S_AB). Since considerations of bar size turns out to be important for understanding reported bar frequencies, I stick with the measurements of Herrera-Endoqui et al. 2015 and treat the "missing" Buta et al. 2015 barred galaxies as unbarred; the bar frequencies I report for $\mathrm{S}^{4} \mathrm{G}$ subsamples may thus be slight underestimates.

As a simple measure of bar "strength" I use the basic subclassifications of bars into strong (SB) and weak (SAB)

1 Buta et al. (2015) noted that improved observing efficiency had allowed the addition of 21 gas-poor galaxies to the original sample. 2 The missing $11 \mathrm{~S} 0$ s are presumably galaxies whose radio velocities were added to the HyperLeda database after the original $\mathrm{S}^{4} \mathrm{G}$ definition in late 2007; see Sheth et al. (2010). 
categories as listed by Buta et al. (2015). Measurements of bar sizes are taken from Herrera-Endoqui et al. (2015). The latter authors provided both "visual" $\left(a_{\mathrm{vis}}\right)$ and maximumellipticity-based $\left(a_{\epsilon}\right)$ measurements of bar lengths (semimajor axes). Díaz-García et al. (2016a) showed that these measurements are generally consistent with each other (e.g., their Fig. 9), with $a_{\epsilon}$ on average marginally smaller than $a_{\text {vis }}$. Since maximum-ellipticity lengths are not available for $\sim 22 \%$ of the bars (usually cases where low S/N and/or strong star formation made ellipse fits unreliable), I use the $a_{\text {vis }}$ values. To compute deprojected sizes, I use bar position angles from Herrera-Endoqui et al. (2015) and disc orientations from Salo et al. (2015).

For galaxy colours, I take $(B-V)_{\text {tc }}$ values from HyperLeda; these are whole-galaxy colours, corrected for Galactic and internal extinction. These are then converted into $g-r$ colours, since that is the most common colour used by published SDSS studies of bar fractions. The colour conversion - and incompleteness corrections necessitated by the fact that about half the galaxies do not have $(B-V)_{\text {tc }}$ colours in HyperLeda - are discussed in more detail in Appendix A.

Finally, I also take $\mathrm{H}$ I fluxes from HyperLeda in order to determine $\mathrm{H}$ I masses and the gas mass ratio $f_{\text {gas }}=M_{\mathrm{HI}} / M_{\star}$. The H I mass is calculated from the HyperLeda $m_{21, \mathrm{c}}$ values using the standard relation of Giovanelli \& Haynes (1988):

$M_{\mathrm{HI}}=2.356 \times 10^{5} D^{2} 10^{0.4\left(17.40-m_{21, \mathrm{c}}\right)}$,

where $D$ is the distance in Mpc and $m_{21, \mathrm{c}}$ is the corrected H I magnitude from HyperLeda. In order to compare bar frequencies as a function of gas mass ratio with the results of Masters et al. (2012), I define $f_{\text {gas }}$ as the ratio of atomic hydrogen to stellar mass, without any corrections for the presence of helium or metals. HyperLeda $m_{21, c}$ values exist for approximately $97 \%$ of the $\mathrm{S}^{4} \mathrm{G}$ galaxies in the Parent Disc Sample (1290 out of 1334 galaxies), so incompleteness is not a meaningful issue. The distributions of $g-r$ and $f_{\text {gas }}$ values as a function of stellar mass in the Parent Spiral Sample are shown in Figure 2.

\subsection{Correcting for Incompleteness: Volume and Mass Limits and Final Sample Definitions}

The original $\mathrm{S}^{4} \mathrm{G}$ sample is magnitude- and diameter-limited (Sheth et al. 2010). The magnitude limit $\left(B_{\mathrm{tc}} \leqslant 15.5\right)$ means that as one moves out in distance, low-luminosity - and thus low-mass - objects drop out of the sample before higherluminosity (higher-mass) objects do. This is demonstrated in Figure 1, which plots stellar mass against distance for each galaxy in the Parent Disc Sample. Distance and stellarmass limits are therefore needed in order to define complete, volume-limited subsamples.

There is an additional reason to apply a more stringent distance limit than the $\sim 40 \mathrm{Mpc}$ cutoff implicit in the original $\mathrm{S}^{4} \mathrm{G}$ sample (defined via a redshift limit of $3000 \mathrm{~km} \mathrm{~s}^{-1}$ ): bars are harder to identify in more distant galaxies. Figure 3 shows observed bar sizes in the Parent Disc Sample, along with lines indicating possible resolution limits as multiples of the $\mathrm{S}^{4} \mathrm{G}$ PSF (assuming FWHM $\approx 2^{\prime \prime}$; Salo et al. 2015). As previously shown by Menéndez-Delmestre et al. (2007) for a study of bars in local galaxies using 2MASS images, physically smaller bars are less likely to be detected at larger distances, because their smaller angular size - combined with
Table 1. $\mathrm{S}^{4} \mathrm{G}$-based Samples

\begin{tabular}{lcrr}
\hline Name & $\begin{array}{r}D_{\max } \\
(\mathrm{Mpc})\end{array}$ & $\begin{array}{r}\text { Minimum } M_{\star} \\
\left(\log \left(M_{\star} / M_{\odot}\right)\right)\end{array}$ & $N$ \\
\hline Parent Disc Sample & - & - & 1322 \\
Parent Spiral Sample & - & - & 1220 \\
Sample 1 & 25 & - & 659 \\
Sample 1m & 25 & 8.5 & 574 \\
Sample 2 & 30 & - & 851 \\
Sample 2m & 30 & 9 & 638
\end{tabular}

Definitions and galaxy counts for different subsamples of $\mathrm{S}^{4} \mathrm{G}$ galaxies used in this paper. Columns: (1) Subsample name. (2) Distance upper limit. (3) Lower limit on stellar mass. (4) Number of galaxies in subsample.

the isophotal-rounding effects of PSF convolution - makes them harder to discern.

Figure 3 suggests that the (visual) bar-detection criteria used by Buta et al. (2015) for the $\mathrm{S}^{4} \mathrm{G}$ sample may start to miss smaller bars for distances $\gtrsim 25-30$ Mpc. Accordingly, I adopt two sets of mass- and distance-limited subsamples. For plots and analyses of bar frequency and size as a function of stellar mass - where the potential incompleteness at the low-mass ends can be visually excluded by the reader - I use distance-limited samples: "Sample 1" assumes a limit of $25 \mathrm{Mpc}$, while "Sample 2" uses $30 \mathrm{Mpc}$. I also construct mass-limited subsamples: Sample $1 \mathrm{~m}$ is Sample 1 with the addition of a stellar-mass limit of $\log \left(M_{\star} / M_{\odot}\right) \geqslant 8.5$; Sample $2 \mathrm{~m}$ is Sample 2 with a higher stellar-mass cutoff of $\log \left(M_{\star} / M_{\odot}\right) \geqslant 9$. These different subsamples are summarized in Table 1, along with the number of galaxies in each. The sample definitions are also indicated by the solid (Samples 1 and $1 \mathrm{~m}$ ) and dashed (Samples 2 and $2 \mathrm{~m}$ ) lines in Figure 1. Figure 4 shows histograms of stellar mass for the Parent Spiral Sample, Sample 1, and Sample 2.

Finally, the diameter limit used to define the original $\mathrm{S}^{4} \mathrm{G}$ sample $\left(D_{25} \geqslant 1.0^{\prime}\right)$ means that there is a distancebased bias against more compact galaxies even after mass limits are applied. The right-hand panel of Figure 1 shows $R_{25}$ in kpc versus galaxy distance for the Parent Spiral Sample. Because this diameter-limit effect is so stark and clean, it makes sense to correct for it using $V / V_{\max }$ weighting. I compute the weights $w$ for each galaxy as

$$
\begin{aligned}
w & =V_{\mathrm{tot}} / V_{\max } \text { if } V_{\max }<V_{\mathrm{tot}}, \\
& =1 \text { otherwise, }
\end{aligned}
$$

where $V_{\text {tot }}$ is the total volume out to the distance limit $D_{\text {lim }}$ (e.g., $D_{\lim }=25 \mathrm{Mpc}$ for Sample 1) and $V_{\max }$ is the volume enclosed by $D_{\max }$, the maximum distance at which the galaxy could still have exceeded the survey's diameter limit. $D_{\text {max }}$ depends on the galaxy's $R_{25}$ in kpc and thus on the observed $R_{25}$ angular size and the galaxy's actual distance $d$; the weights can thus be expressed as

$$
\begin{aligned}
w & =\left(D_{\max }^{3} R_{25, \lim }\right) /\left(d^{3} R_{25}\right) \text { if } D_{\max }<D_{\lim } \\
& =1 \text { otherwise }
\end{aligned}
$$

where $R_{25, \lim }=30^{\prime \prime}$. 

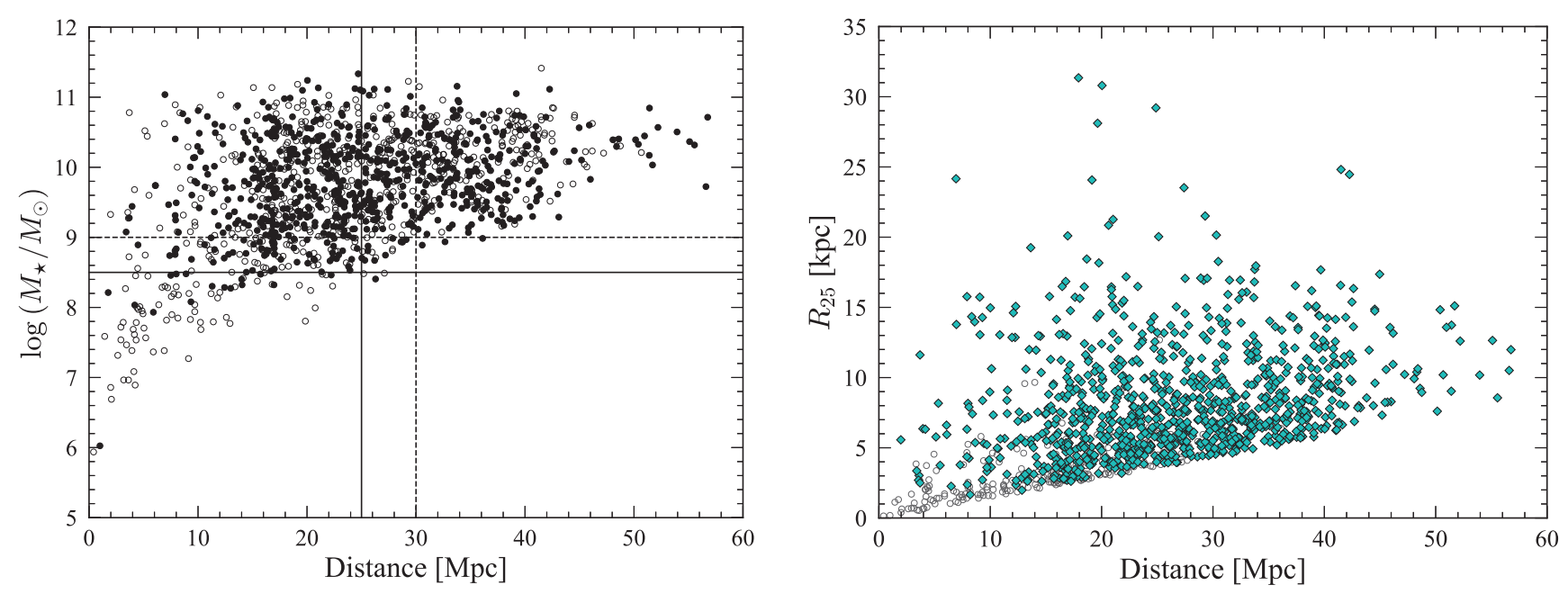

Figure 1. Effects of magnitude and angular-size limits on stellar mass and absolute disc size for the $S^{4} G$ galaxies in the Parent Disc Sample, based on the low-inclination $\mathrm{S}^{4} \mathrm{G}$ disc sample of Díaz-García et al. (2016a). (Five galaxies with $D>60$ Mpc are not shown.) Left: Stellar mass versus distance. Hollow points are unbarred galaxies, filled points are barred. Lines show distance and stellar-mass cuts used to define the main volume-limited subsamples for this paper (see Table 1). Right: $B$-band optical disc radius $R_{25}$ versus distance (spiral galaxies only). Cyan diamonds indicate galaxies with stellar masses $\log \left(M_{\star} / M_{\odot}\right) \geqslant 9$.
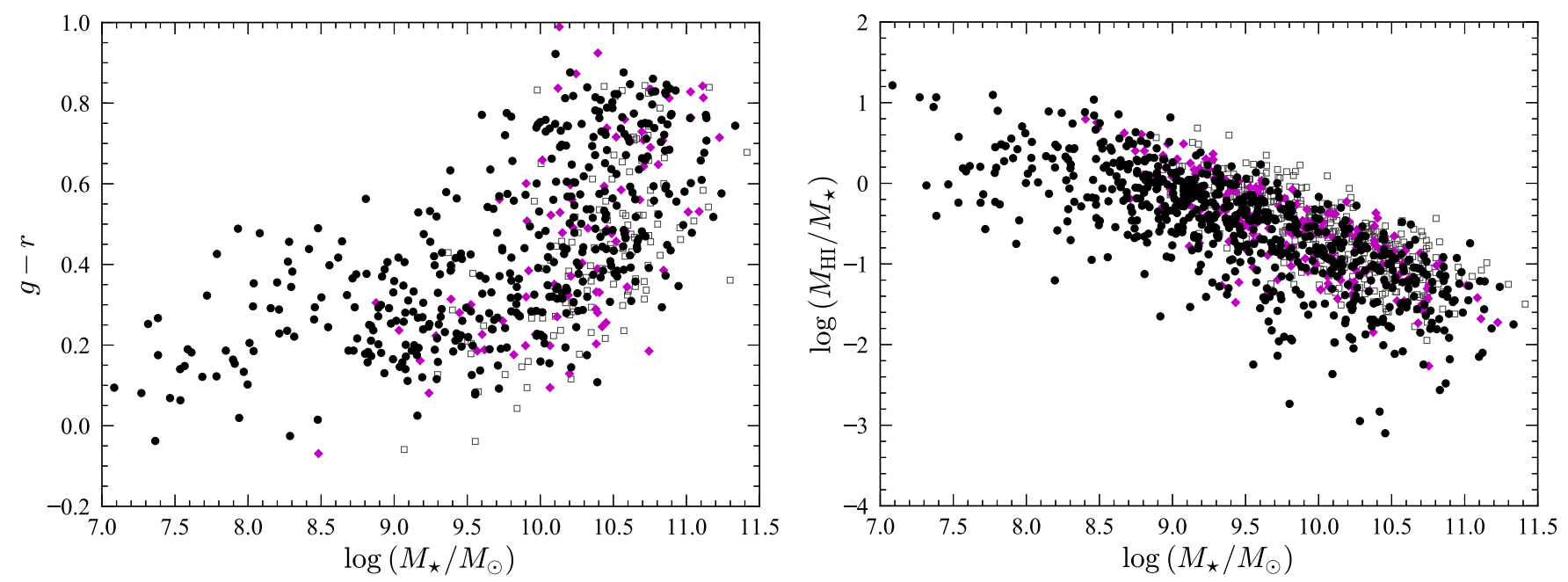

Figure 2. Galaxy $g-r$ colours (left) and gas mass fraction $f_{\text {gas }}$ (right) for $\mathrm{S}^{4} \mathrm{G}$ spiral galaxies. Galaxies with distances $\leqslant 25 \mathrm{Mpc}$ are plotted with filled black circles; galaxies with distances between 25 and $30 \mathrm{Mpc}$ are plotted with filled magenta diamonds, while galaxies at larger distances (not analyzed in this paper) are plotted with open squares.

\section{BAR FRACTIONS FOR LOCAL GALAXIES}

The bar fractions for $\mathrm{S}^{4} \mathrm{G}$ galaxies from Samples 1 or $1 \mathrm{~m}$ are shown in Figure 5, first for for all bars (red circles, left panels) and then for for strong and weak bars considered separately (black and cyan circles, right panels). I show these fractions as a function of three galaxy parameters: stellar mass (top panels), $g-r$ colour (middle panels), and gas mass ratio $f_{\text {gas }}$ (bottom panels). The error bars attached to each bar fraction account for the $V / V_{\max }$ weights (and $B_{\text {tc }}$-based weights for the $g-r$ plots) using the approach of Wilman \& Erwin (2012): all weighted counts in a given bin were rescaled so that the total was equal to the original unweighted total counts for that bin, and these were then used to estimate the Wilson (1927) 68\% confidence limits.
Additional bar-fraction estimates from a subset of different SDSS-based studies are shown in the left-hand panels; see below for the specific studies.

\subsection{Bar Fraction and Galaxy Stellar Mass}

The upper left panel of Figure 5 shows how the bar fraction $f_{\text {bar }}$ in $\mathrm{S}^{4} \mathrm{G}$ galaxies varies with stellar mass. There is a steep increase from very low stellar masses, reaching a maximum of $f_{\text {bar }}=0.76$ at $\log \left(M_{\star} / M_{\odot}\right) \sim 9.8$, and then a similarly steep decline to higher masses. Although Sample 1 is incomplete for $\log \left(M_{\star} / M_{\odot}\right) \lesssim 8.5$, this is a volume effect and should not affect the detectability of bars in lower-mass galaxies; it only reduces the total number of galaxies and 


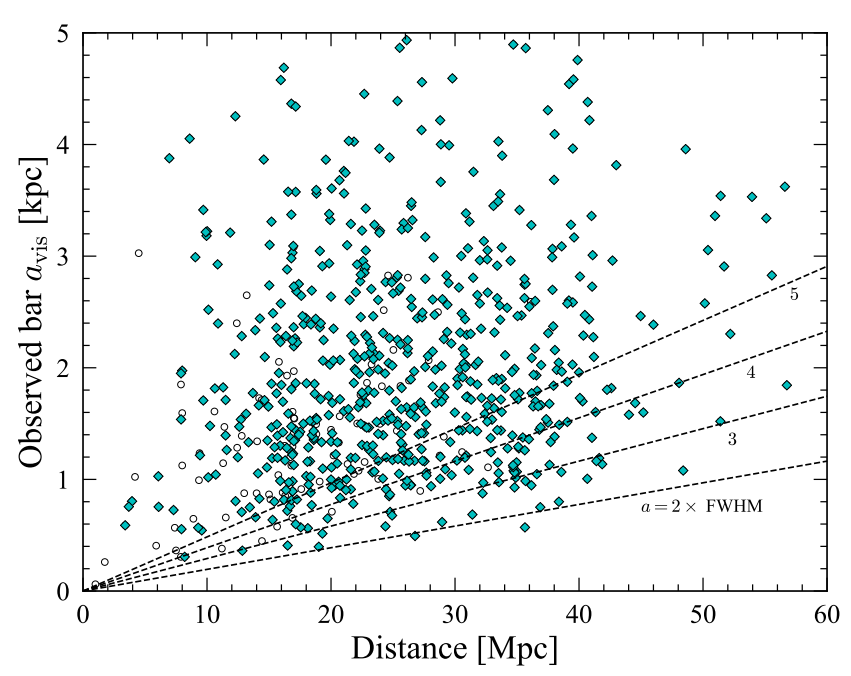

Figure 3. Observed bar sizes (semi-major axes) in Parent Disc Sample (i.e., including S0 galaxies) versus galaxy distance. Dashed lines show possible resolution limits in multiples of the $\mathrm{S}^{4} \mathrm{G}$ PSF's FWHM $\left(\approx 2^{\prime \prime}\right)$. Cyan diamonds are galaxies with $\log \left(M_{\star} / M_{\odot}\right) \geqslant 9$.

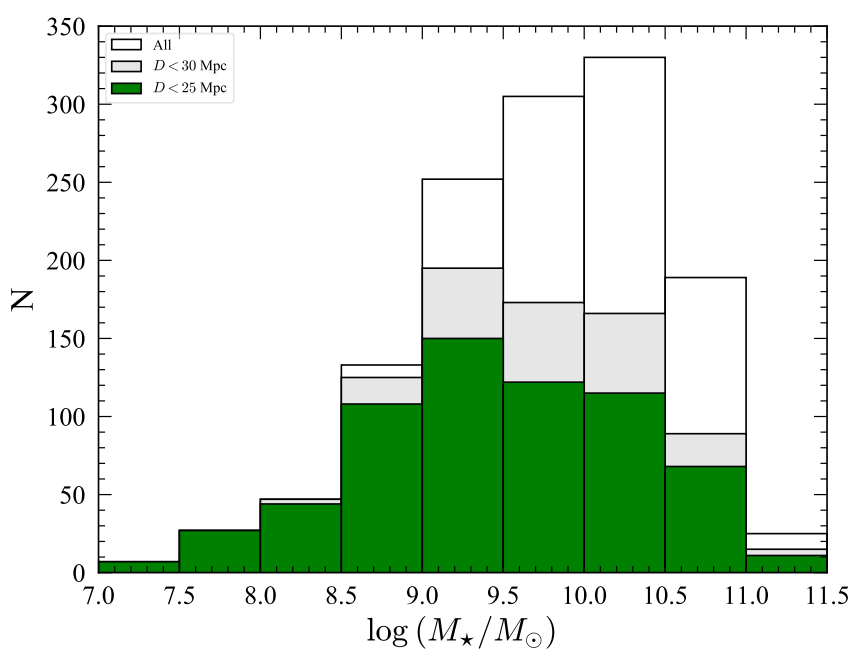

Figure 4. Histograms of stellar mass for different distancelimited subsamples of the $\mathrm{S}^{4} \mathrm{G}$ Parent Spiral Sample.

makes the bar fraction more uncertain. Thus, the steep decline in $f_{\text {bar }}$ seen for $\log \left(M_{\star} / M_{\odot}\right) \lesssim 8.5$ is probably real.

This pattern is very similar to the "visual" $f_{\text {bar }}$ trend in Díaz-García et al. (2016a, their Fig. 19; see also Appendix B), since both are based on the Buta et al. (2015) bar classifications for $\mathrm{S}^{4} \mathrm{G}$. The main difference is their shallower slope for higher masses $\left(\log \left(M_{\star} / M_{\odot}\right) \gtrsim 10\right)$ and the existence of a secondary peak in $f_{\text {bar }}$ at $\log \left(M_{\star} / M_{\odot}\right) \sim 10.5$. These differences are (necessarily) due to the fact that Sample 1 is a distance-limited subset of the Parent Disc Sample, and the fact that it excludes S0 galaxies. For example, adding the S0s to Sample 1 produces a flatter trend of $f_{\text {bar }}$ versus $\log \left(M_{\star} / M_{\odot}\right)$ for higher stellar masses (see Appendix B2).

In an attempt to parameterize the trend - and to avoid dependencies on the binning used for the figure - I also plot the results of a polynomial logistic regression applied to Sample 1 (dashed red line). Logistic regression involves modeling the presence or absence of a feature (in this case, bars) using a function which represents the binomial probability of that feature. It has the advantage of using all the data directly rather than relying on the details of a binning scheme. A generalized polynomial version (of order $n$ ) is

$f_{\mathrm{bar}}(x)=\frac{1}{1+e^{-\left(\alpha+\sum_{i=1}^{n} \beta_{i} x^{i}\right)}}$,

where $x$ is the independent variable (e.g., $\log \left(M_{\star} / M_{\odot}\right)$ ). Although logistic regression typically involves a linear function of $x$, leading to a probability that either monotonically increases or decreases in a sigmoid fashion, the behavior of $f_{\text {bar }}$ is clearly not monotonic, so a quadratic function $(n=2)$ is probably better. The result (with bestfit coefficients $\alpha=-82.2 \pm 22.1, \beta_{1}=17.1 \pm 4.62$, and $\left.\beta_{2}=-0.88 \pm 0.24\right)$ is plotted as a dashed red line in Figure 5; the peak in $f_{\text {bar }}$ is $\approx 0.70$ at $\log \left(M_{\star} / M_{\odot}\right) \approx 9.7$. (The fitting was done to data from Sample 1 from $\log \left(M_{\star} / M_{\odot}\right)=8-12$ with $V / V_{\max }$-based weights, using the R Survey package. ${ }^{3}$ )

Also shown in the upper left panel of Figure 5 are the reported trends for GZ2 from Masters et al. (2012) and (for a larger sample) from Melvin et al. (2014). Both of these studies suggest a very low $f_{\text {bar }}$ for $\log \left(M_{\star} / M_{\odot}\right) \lesssim 10$ and a steep increase in $f_{\text {bar }}$ for higher stellar masses, with a maximum at $\log \left(M_{\star} / M_{\odot}\right) \sim 10.8$. A similar trend can be seen in the non-GZ2 SDSS-based results of Gavazzi et al. 2015. This clearly does not agree with the $\mathrm{S}^{4} \mathrm{G}$ values; indeed, for $\log \left(M_{\star} / M_{\odot}\right) \sim 10-11$, the $\mathrm{S}^{4} \mathrm{G}$ and SDSS-based bar frequencies show the opposite behavior. The more complicated dependence seen by Nair \& Abraham (2010b) is not apparent in the $\mathrm{S}^{4} \mathrm{G}$ data, either.

There is rather good agreement with the results of Barazza et al. (2008, blue diamonds) ${ }^{4}$ and fairly good agreement with Méndez-Abreu et al. (2012, not shown), especially with the latter's "Field" subsample, where $f_{\text {bar }}$ peaks at $\log \left(M_{\star} / M_{\odot}\right) \sim 9.5 ; f_{\text {bar }}$ for their "Virgo" subsample peaks at $\log \left(M_{\star} / M_{\odot}\right) \sim 10.1$. Since the Virgo subsample is at distances $<20 \mathrm{Mpc}$, and since most of the field subsample was defined with redshifts between 2500 and $3000 \mathrm{~km} \mathrm{~s}^{-1}$, the spatial resolution of the SDSS images they use is generally similar to that of the $\mathrm{S}^{4} \mathrm{G}$ sample.

The upper right panel of Figure 5 shows the bar fraction separately for strong (SB) and weak (SAB) bars. The SAB fraction is almost constant for $\log \left(M_{\star} / M_{\odot}\right) \sim 8.5-10.7$, declining slightly to both lower and higher masses. Strong bars show a more dramatic version of the global bar trend: $f_{\text {bar }}$ increases from $\sim 0$ at the lowest stellar masses to a rather sharp peak at $\log \left(M_{\star} / M_{\odot}\right) \approx 9.8$, and then falls off steeply

3 http://r-survey.r-forge.r-project.org/survey/index. html

${ }^{4}$ For the Barazza et al. (2008) results here and in the left middle panel, I use their per-bin total-galaxy counts to estimate proper binomial uncertainties; this is generally not possible for the other SDSS-based studies. 
to higher masses. This suggests that the clear dependence of $f_{\text {bar }}$ on stellar mass is driven primarily by strong bars.

\subsection{Bar Fraction and Galaxy Colour}

The middle panels of Figure 5 shows the fraction of $\mathrm{S}^{4} \mathrm{G}$ galaxies in Sample $1 \mathrm{~m}$ with bars, as a function of $g-r$ colour, ${ }^{5}$ for all bar types (left panel) and separately for strong and weak bars (right panel). The overall relation is fairly flat, although it is possible to make out a weak trend of $f_{\text {bar }}$ declining to redder colours. The dashed red line shows a standard (linear) logistic regression to the underlying data; it indicates a declining bar fraction to redder colours, though the nonzero slope is not statistically significant. Figure A2 (in Appendix A2) shows almost identical behavior when $B-$ $V$ colour is used instead of $g-r$.

I also plot the published GZ2 trends from Masters et al. (2011) and Masters et al. (2012) and the non-GZ2 trend from Lee et al. (2012a) in the middle left panel (grey symbols). These disagree strongly with the $\mathrm{S}^{4} \mathrm{G}$ trend, particularly given the steep rise in GZ2 $f_{\text {bar }}$ to redder colours $(g-r \sim 0.5-0.75)$.

The trend of $f_{\text {bar }}$ versus colour from Nair \& Abraham (2010b, cyan stars) is similar to the GZ2 trend for intermediate colours $(g-r \sim 0.3-0.65)$, but diverges for the reddest colours. It, too, agrees poorly with the $\mathrm{S}^{4} \mathrm{G}$ trend.

In contrast to the other SDSS-based studies, the Barazza et al. (2008) trend (blue diamonds) shows extremely good agreement with the $\mathrm{S}^{4} \mathrm{G}$ results, including the slight decrease in $f_{\text {bar }}$ to redder colours.

The separate trends for strong and weak bars (righthand middle panel) are, within the uncertainties, indistinguishable and roughly constant.

\subsection{Bar Fraction and Atomic Gas Fraction}

In the lower left panel of Figure 5 I plot $f_{\text {bar }}$ as a function of the atomic gas mass ratio $f_{\text {gas }}$. The $\mathrm{S}^{4} \mathrm{G}$ bar fraction is basically constant, apart from a weak minimum at $\log f_{\text {gas }} \sim-1.3$. The dashed red line shows a standard (linear) logistic regression to the underlying data; the slope is not statistically significant, so there is no evidence for a change in $f_{\text {bar }}$ with $\log f_{\text {gas }}$.

Plotted in the same panel is the GZ2 trend reported by Masters et al. (2012), which shows fairly strong disagreement: the GZ2 $f_{\text {bar }}$ value drops steeply for $\log f_{\text {gas }}$ between -1.0 and 0 , while for $\mathrm{S}^{4} \mathrm{G}$ galaxies $f_{\text {bar }}$ reaches a (weak) local maximum within the same range, and shows no sign of a systematic decline. Also shown are the reported $\log f_{\text {gas }}$ values from the SDSS-based study of Cervantes Sodi (2017), ${ }^{6}$ which show a pattern very similar to the GZ2 one.

The lower right panel of Figure 5 shows how the frequencies of strong and weak bars behave as function of gas mass ratio. For values of $\log f_{\text {gas }}$ between -2 and $0.5\left(f_{\text {gas }}\right.$ between $\sim 0.01$ and 0.3 ), the fractions of both strong and weak bars are essentially identical - and constant. This may

5 Corrected for colour incompleteness as described in Section A1.

6 These have had 0.146 dex subtracted to account for the fact that Cervantes Sodi scaled the H I gas masses by a factor of 1.4 in order to include helium. also be true for very low gas fractions, though the small numbers make the fractions uncertain. Only for very high gas fractions $\left(f_{\text {gas }} \gtrsim 1\right)$ is there a noticeable difference: the fraction of weak bars increases, while the fraction of strong bars goes down.

It is important to note here that the SDSS-based studies are not directly comparable in terms of sample definition. Specifically, the sample of Masters et al. (2012) was defined using $\mathrm{H}$ I detections from the $\alpha 40$ release of Arecibo Legacy Fast ALFA (ALFALFA) survey (Giovanelli et al. 2005; Haynes et al. 2011). Only $51 \%$ of the GZ2 galaxies observed as part of ALFALFA were detected in $\mathrm{H}$ i; the resulting gas mass fractions are relatively high (median $f_{\text {gas }}=0.39$ for their barred galaxies and 0.74 for the more numerous unbarred galaxies). This contrasts strongly with the $\sim 97 \% \mathrm{H}$ I detection rate for the $\mathrm{S}^{4} \mathrm{G}$ sample; the median value of $f_{\text {gas }}$ for the $\mathrm{S}^{4} \mathrm{G}$ galaxies is 0.25 . (If we match the GZ2 sample more closely by only considering $\mathrm{S}^{4} \mathrm{G}$ galaxies with $\log \left(M_{\star} / M_{\odot}\right) \geqslant 9$, then the $\mathrm{S}^{4} \mathrm{G}$ median is even lower: $f_{\text {gas }}=0.19$.) Similar considerations undoubtedly apply to the $f_{\text {bar }}$ versus $f_{\text {gas }}$ analysis of Cervantes Sodi (2017), since that study used an expanded version of the same ALFALFA $\mathrm{H}$ I observations and a similar set of SDSS galaxies.

Since the $S^{4} G$ sample is far more complete in terms of $\mathrm{H}$ I detections, and samples down to much lower values of $f_{\text {gas }}$, than is true for the SDSS studies, it is probably more representative in terms of how bars do (or do not) depend on the gas mass fraction in galaxies.

\subsection{Strong Bars versus Weak Bars}

Several SDSS-based papers have argued that their detections are primarily of "strong" bars. For example, by comparing galaxies in common between GZ2 and the sample of Nair \& Abraham (2010b), Masters et al. (2012) suggested that galaxies with GZ2 $p_{\text {bar }} \geqslant 0.5$ - their standard definition for a galaxy being barred - were equivalent to the "strong bars" of Nair \& Abraham (2010a,b). Is there any evidence that local $\left(\mathrm{S}^{4} \mathrm{G}\right)$ galaxies behave more like GZ2 and other SDSS galaxies when only strong bars are considered?

Figure 5 suggests that strong (SB) and weak (SAB) bars in $\mathrm{S}^{4} \mathrm{G}$ behave almost indistinguishably, with two exceptions. First, strong bars display a more sharply peaked distribution as a function of stellar mass, with a maximum at $\log \left(M_{\star} / M_{\odot}\right) \sim 9.8$, in contrast to the very broad maximum in $f_{\text {bar }}$ seen for $\mathrm{SAB}$ bars (upper right panel of Figure 5). Second, the fractions of $\mathrm{SB}$ and $\mathrm{SAB}$ bars $\left(f_{\mathrm{SB}}\right.$ and $\left.f_{\mathrm{SAB}}\right)$ diverge for very high gas mass ratios: for $f_{\text {gas }}>1, f_{\mathrm{SB}}$ decreases and $f_{\mathrm{SAB}}$ increases. But neither of these SB trends agrees with the general SDSS-based results. Indeed, the $f_{\mathrm{SB}}$ trend as a function of stellar mass is if anything even more different from the SDSS-based trends than is true for $f_{\text {bar }}$.

Similarly, the fact that the $\mathrm{S}^{4} \mathrm{G} f_{\mathrm{SB}}$ and $f_{\mathrm{SAB}}$ values remain roughly constant (and equal) for $\log f_{\text {gas }}<0$ (lower right panel of Figure 5) means that the strong change in $f_{\text {bar }}$ versus $\log f_{\text {gas }}$ seen by Masters et al. (2012) and Cervantes Sodi (2017) (over the range $f_{\text {gas }} \approx-1.0$ to 0 ) cannot be due to their detecting only strong bars. Finally, the trends of $f_{\mathrm{SB}}$ and $f_{\mathrm{SAB}}$ as a function of $g-r$ (middle right panel of Figure 5) are identical within the uncertainties, with both indicating either no correlation with colour or else a slight decrease in bar fraction towards redder colours. 

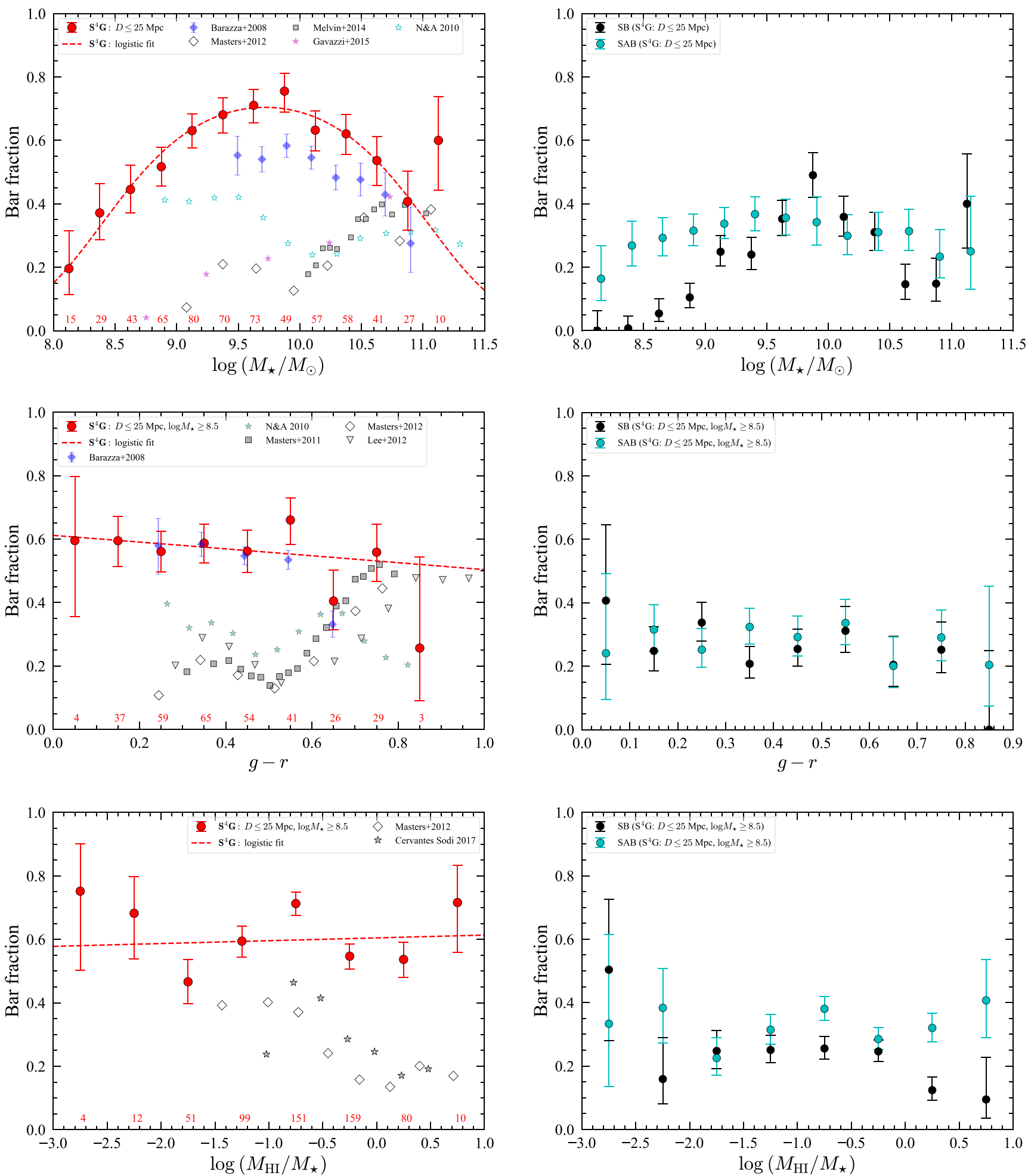

Figure 5. Fractions of disc (spiral + Irr) galaxies with bars (left panels) or with strong (SB) and weak (SAB) bars counted separately (right panels). Fractions for $\mathrm{S}^{4} \mathrm{G}$ samples are shown with red circles (left panels) or black and green circles (right panels); dashed red lines show the results of logistic fits to the underlying data; see text for details. The small red numbers along the bottom of each left-hand panel are the raw $\mathrm{S}^{4} \mathrm{G}$ counts per bin. Top panels: Bar fractions as a function of stellar mass for $\mathrm{S}^{4} \mathrm{G}$ galaxies with distances $\leqslant 25$ Mpc. Other symbols show reported bar fractions for GZ2 (Masters et al. 2012; Melvin et al. 2014) and three other SDSS-based studies (Barazza et al. 2008; Nair \& Abraham 2010b; Gavazzi et al. 2015). Middle panels: Bar fractions as a function of $g-r$ colour for $S^{4} \mathrm{G}$ galaxies in Sample $1 \mathrm{~m}$ (based on $B-V$ colours). Gray squares and diamonds show reported bar fractions for GZ2 (Masters et al. 2011, 2012), with grey triangles for Lee et al. (2012a) and cyan stars for Nair \& Abraham (2010b). Bottom panels: Bar fractions as a function of atomic gas fraction $f_{\text {gas }}$ for $\mathrm{S}^{4} \mathrm{G}$ galaxies in Sample 1m. Also plotted are reported SDSS-based trends for GZ2 (Masters et al. 2012, open diamonds) and Cervantes Sodi (2017, gray stars). 
Table 2. Total Bar Fractions

\begin{tabular}{lcrlll}
\hline Name & $\begin{array}{c}D_{\max } \\
(\mathrm{Mpc})\end{array}$ & $\begin{array}{c}\text { Minimum } M_{\star} \\
\left(\log \left(M_{\star} / M_{\odot}\right)\right)\end{array}$ & $f_{\text {bar }}$ & $f_{\mathrm{SB}}$ & $f_{\mathrm{SAB}}$ \\
\hline Sample 1 & 25 & - & $0.563 \pm 0.019$ & $0.234_{-0.016}^{+0.017}$ & $0.329 \pm 0.018$ \\
Sample 1m & 25 & 8.5 & $0.618 \pm 0.020$ & $0.267_{-0.018}^{+0.019}$ & $0.351 \pm 0.020$ \\
Sample 2 & 30 & - & $0.564 \pm 0.017$ & $0.236_{-0.014}^{+0.015}$ & $0.328 \pm 0.016$ \\
Sample 2m & 30 & 9 & $0.623 \pm 0.019$ & $0.290 \pm 0.018$ & $0.333_{-0.018}^{+0.019}$ \\
\hline
\end{tabular}

Observed bar fractions for different subsamples of $\mathrm{S}^{4} \mathrm{G}$ spiral galaxies. For each subsample, I list the total bar fraction $f_{\text {bar }}$ and the separate fractions for strong (SB) and weak (SAB) bars. The fractions for Samples 1 and 2 include all galaxies within the specified distance limits; the fractions for Samples $1 \mathrm{~m}$ and $2 \mathrm{~m}$ are restricted to galaxies more massive than the specified stellar-mass limits.

So it appears that we cannot explain the discrepancies between the $\mathrm{S}^{4} \mathrm{G}$-based results and the SDSS-based results by appealing to the hypothesis that the latter are simply the behavior of strong bars rather than bars in general.

It should be kept in mind that the concept of "strong" versus "weak" bars is inherently a somewhat ambiguous one. Various quantitative measures of bar strength have been proposed - and several of them have been applied to many (though not all) of the bars in $\mathrm{S}^{4} \mathrm{G}$ - but they can sometimes be surprisingly contradictory, in part because they aim at defining different aspects of "strength". Figs. 17, 18, and 20 of Díaz-García et al. (2016a) shows some of the potential ambiguity for the $\mathrm{S}^{4} \mathrm{G}$ galaxies. For example, if one chooses the $A_{2 \text {,max }}$ measure often used by theorists, then there is fairly clear correlation (albeit with considerable scatter) between galaxy mass and bar strength: on average, the strongest bars are in high-mass galaxies. If one uses the maximum isophotal ellipticity (probably the easiest observational measurement), then there is no correlation. For $Q_{b}$ (the maximum gravitational torque produced by the bar), there is an anticorrelation, with the strongest bars in lower-mass galaxies. ${ }^{7}$ Similarly, if one follows Lee et al. (2012a) and Cervantes Sodi (2017) and uses the size of the bar relative to the apparent or fiducial size of the disc, then the strongest bars would be in the lowest-mass galaxies (see also Figure 9). In general, one should probably be cautious about claims that SDSSbased studies are detecting mostly (or only) "strong bars", since unless the type of bar strength is carefully specified, the implications are unclear.

\subsection{The Global Bar Fraction}

As demonstrated in the preceding subsections, the bar fraction $f_{\text {bar }}$ is roughly constant with both colour and gas mass ratio, but is a strong function of stellar mass. The latter fact means that there is no one "global" bar fraction, since the fraction for any sample will depend on the masses of the galaxies in the sample. With that caveat in mind, I present summary fractions in Table 2, which shows the bar fraction for the different $\mathrm{S}^{4} \mathrm{G}$ spiral subsamples. The bar fraction for all spirals is $0.56 \pm 0.02$; however, this is probably a slight overestimate given the incompleteness in galaxies with

7 This is at least partly because $Q_{b}$ measures the gravitational strength of the bar relative to the bulge and inner disk; in lowmass galaxies with little or no bulge, the bar's impact is less diluted and so $Q_{b}$ is stronger. $\log \left(M_{\star} / M_{\odot}\right)<9$, since low-mass galaxies are less likely to have bars. For spirals with $\log \left(M_{\star} / M_{\odot}\right) \gtrsim 9$, the fraction is $0.62 \pm 0.02$. This is consistent with most previous estimates from smaller local samples (e.g., Sellwood \& Wilkinson 1993; Mulchaey \& Regan 1997; Knapen et al. 2000; Eskridge et al. 2000; Menéndez-Delmestre et al. 2007; and the discussion in Sheth et al. 2008), which tended to be dominated by highmass galaxies.

\section{THE SIZES AND DETECTABILITY OF BARS}

\subsection{Why Do $S^{4} \mathrm{G}$ Bar Frequencies Differ From (Most) SDSS Results?}

The previous section has shown that the frequencies of bars in local $\left(\mathrm{S}^{4} \mathrm{G}\right)$ galaxies generally differ markedly from those reported for most SDSS-based samples - not just in overall frequency, but also in trends with stellar mass, colour, and gas fraction. Why is this so?

It seems unlikely that cosmic variance could produce such a strong, systematic difference. The fact that the Barazza et al. (2008) and Méndez-Abreu et al. (2012) studies actually agree rather rather well with the local results makes such an appeal even more improbable. Similarly, while the use of near-IR images for the $\mathrm{S}^{4} \mathrm{G}$ sample largely eliminates the possibility of missing bars due to strong dust extinction or star formation, which might affect SDSS optical images, it is unclear why dust and star formation should not affect the Barazza et al. (2008) or Méndez-Abreu et al. (2012) results in the same way as most other SDSS-based studies. Differences in bar-detection methods could certainly explain some of the difference, particularly since some methods may be more sensitive to weaker or smaller bars than others. Still, SDSS-based studies with different bar-detection methods do show similar results: e.g., GZ2 (Masters et al. 2011, 2012; Skibba et al. 2012), Oh et al. (2012), and Lee et al. (2012a) for $f_{\text {bar }}$ versus $g-r$; GZ2 (Masters et al. 2012; Skibba et al. 2012; Melvin et al. 2014), Oh et al. (2012), Gavazzi et al. (2015), and Cervantes Sodi (2017) for $f_{\text {bar }}$ versus stellar mass; or GZ2 and Cervantes Sodi (2017) for $f_{\text {bar }}$ versus gas mass fraction. So it is hard to see how the strong differences between most SDSS-based studies and the local, $\mathrm{S}^{4} \mathrm{G}$ results can be explained this way.

One key difference between the $\mathrm{S}^{4} \mathrm{G}$ galaxies and the SDSS samples is spatial resolution. Although the FWHM for the Spitzer $3.6 \mu \mathrm{m}$ images used for $\mathrm{S}^{4} \mathrm{G}\left(\approx 2^{\prime \prime}\right)$ is slightly 
worse than the typical SDSS seeing of $\sim 1.4^{\prime \prime}$, SDSS galaxies are, on average, much further away, so the effective linear resolution is correspondingly much poorer. The mean distance of $\mathrm{S}^{4} \mathrm{G}$ galaxies in Sample 1 is $16.8 \mathrm{Mpc}$ (median = $17.8 \mathrm{Mpc}$ ), so the FWHM translates to a mean linear resolution of $\sim 165$ pc. For a volume-limited sample spanning $z=0.01-0.06$, as used by most of the GZ2 studies, the mean redshift would be $\approx 0.045$ (assuming a uniform galaxy density), with a mean linear resolution of $\approx 1.25 \mathrm{kpc}-$ almost an order of magnitude worse.

In this context, it is significant that the SDSS-based studies which do show agreement with the local galaxies that is, Barazza et al. (2008) and Méndez-Abreu et al. (2012) - are samples of galaxies at smaller distances. The Barazza et al. (2008) sample had a redshift range of $z=0.01-0.03$, which implies (assuming uniform densities) $\langle z\rangle \approx 0.023$ and a mean spatial resolution twice as good as GZ2 and similar SDSS-based studies. The "field2" subsample of MéndezAbreu et al. (2012) used galaxies with redshifts of 2500-3000 $\mathrm{km} \mathrm{s}^{-1}$, their "Virgo" subsample used Virgo Cluster galaxies, and their "Coma" subsample used HST rather than SDSS images, so their spatial resolutions were similar to or slightly better than that of the $\mathrm{S}^{4} \mathrm{G}$ galaxies.

Poorer resolution has the obvious effect of reducing the observed ellipticity and contrast of a bar (perhaps especially so if there is a bright bulge whose light can be convolved with that of the bar), and this will be worse for bars which are smaller in angular size. Figure 3 suggests a possible limit of $\sim 2 \times$ FWHM for detecting bars in $\mathrm{S}^{4} \mathrm{G}$. Although there is a potential complication due to the existence of an angular size limit for the parent $S^{4} G$ sample - e.g., at larger distances galaxies with smaller optical discs are preferentially excluded, which may in turn exclude smaller bars - Aguerri et al. (2009) concluded from their experiments with artificial galaxy images that a lower limit on detectable bar size for SDSS images was $a_{\text {bar }} \sim 3.6^{\prime \prime}$, or about $2.5 \times$ FWHM.

In the context of GZ2, Willett et al. (2013) explicitly pointed out a possible bias against detecting smaller bars, based both on the GZ2 public interface ("GZ2 participants may not have looked for bars shorter than the disc diameter, or have been less confident in voting for 'yes' if they were identified") and also on a strong correlation between the GZ2 $p_{\text {bar }}$ parameter and the EFIGI (Baillard et al. 2011) bar "length" parameter for galaxies in common between GZ2 and EFIGI.

So - despite arguments sometimes made that all "normal" or "galactic-scale" bars can be detected in SDSS samples - it is at least possible that most SDSS studies are failing to detect significant numbers of bars due to resolution effects. (Some SDSS-based studies - e.g., Gadotti 2011; Wang et al. 2012 - are careful to point out that they are less sensitive to smaller bars.) Whether this might explain the different systematics in bar frequency, depends on, among other things, the actual sizes of bars - and whether their sizes vary significantly with galaxy properties such as mass, colour, or gas content.

\subsection{The Sizes of Bars in $\mathrm{S}^{4} \mathrm{G}$}

Figure 6 shows how bar size behaves as a function of stellar mass for $\mathrm{S}^{4} \mathrm{G}$ galaxies in Samples 1 and 2. There are two important things to note about this figure.

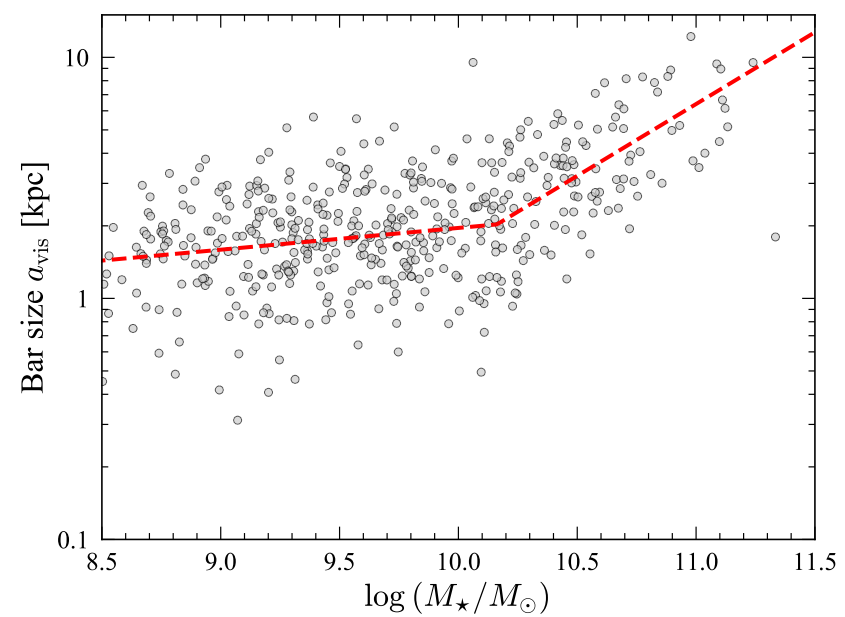

Figure 6. Bar semi-major axis versus stellar mass for $\mathrm{S}^{4} \mathrm{G}$ spiral galaxies with $D \leqslant 25 \mathrm{Mpc}$ (dark circles); galaxies with distances between 25 and $30 \mathrm{Mpc}$ are plotted with open circles. The red dashed line shows a broken linear fit to galaxies with $D \leqslant 30$ Mpc and $\log \left(M_{\star} / M_{\odot}\right)=9-11$; see Paper II for more details.

The first is that there is considerable scatter in intrinsic bar size for any given stellar mass; among other things, this scatter means that a significant fraction of bars have semimajor axes $<1 \mathrm{kpc}$. Although many studies of bars in SDSS samples correctly note that bars with sizes $\lesssim 1 \mathrm{kpc}$ will probably go undetected in SDSS images, they often argue that bars of this size are "nuclear bars" distinct from "normal" bars, and thus any failures to reliably detect bars of this size do not affect conclusions about bars (or normal bars) in general. But as Figure 6 demonstrates, sub-kpc bars appear to be simply the tail end of the general distribution of bar sizes for galaxies with masses $\log \left(M_{\star} / M_{\odot}\right) \lesssim 10.2$, and so failing to detect such bars will bias conclusions about bar frequencies. Only for galaxies with masses $\log \left(M_{\star} / M_{\odot}\right) \gtrsim 10.3$ could one argue that bars with lengths $<1 \mathrm{kpc}$ would be different from the general population of bars.

The second point is that there is indeed, as noted by Díaz-García et al. (2016a, see their Fig. 20 and Table 5), a clear correlation between bar size and stellar mass. Closer inspection suggests the trend actually has a bimodal quality: bar size is almost constant (but increases slightly with stellar mass) for stellar masses $\lesssim 10^{10} M_{\odot}$, and clearly increases with stellar mass for higher masses.

Further analysis of the correlation between bar size and stellar mass (and other galaxy properties) will be presented in Erwin (2018, in prep; hereafter Paper II). Here, I emphasize the basic size-mass correlation, as well as the general observation that a correlation between bar size and stellar mass will probably translate into a correlation between bar size and galaxy colour and an anticorrelation between bar size and gas content (since more massive galaxies tend to redder and more gas-poor; see Figure 2). Evidence for this (especially for the gas-content-bar-size anticorrelation) can be seen in Figure 7. 
Table 3. Fits to Bar Size versus Stellar Mass

\begin{tabular}{rcccccc}
\hline Fit range & $D_{\max }$ & $\alpha_{1}$ & $\beta_{1}$ & $\alpha_{2}$ & $\beta_{2}$ & $\log \left(M_{\mathrm{brk}} / M_{\odot}\right)$ \\
$\left(\log \left(M_{\star} / M_{\odot}\right)\right)$ & $(\mathrm{Mpc})$ & & & & & \\
$(1)$ & $(2)$ & $(3)$ & $(4)$ & $(5)$ & $(6)$ & $(7)$ \\
\hline 8.5 & 25 & $-0.62_{-0.22}^{+0.36}$ & $0.09_{-0.04}^{+0.02}$ & $-4.35_{-0.55}^{+1.08}$ & $0.46_{-0.10}^{+0.05}$ & $10.01_{-0.16}^{+0.04}$ \\
$8.5-11$ & 25 & $-0.75_{-0.30}^{+0.26}$ & $0.10_{-0.03}^{+0.03}$ & $-5.80_{-0.92}^{+0.87}$ & $0.60_{-0.08}^{+0.09}$ & $10.16_{-0.07}^{+0.08}$ \\
$\geqslant 9$ & 30 & $-0.69_{-0.52}^{+0.28}$ & $0.10_{-0.03}^{+0.05}$ & $-4.56_{-0.56}^{+0.72}$ & $0.48_{-0.07}^{+0.05}$ & $10.10_{-0.06}^{+0.09}$ \\
$9-11$ & 30 & $-0.53_{-0.38}^{+0.41}$ & $0.08_{-0.04}^{+0.04}$ & $-5.39_{-0.47}^{+1.13}$ & $0.56_{-0.11}^{+0.05}$ & $10.11_{-0.13}^{+0.02}$ \\
All & - & $-1.17_{-0.31}^{+0.22}$ & $0.15_{-0.02}^{+0.03}$ & $-4.17_{-0.66}^{+0.61}$ & $0.44_{-0.06}^{+0.06}$ & $10.24_{-0.03}^{+0.07}$ \\
\hline
\end{tabular}

Results of broken-linear fits to $\log a_{\mathrm{vis}}$ as a function of $\log \left(M_{\star} / M_{\odot}\right)$. Parameter uncertainties are based on 2000 rounds of bootstrap resampling.

\subsection{Implications for SDSS-Based Surveys}

The implication of the bar-size-stellar-mass correlation is this: because higher-mass galaxies tend to have physically larger bars, their bars will be easier to detect at large distances than is true for bars in lower-mass galaxies. If the resolution limit of a survey is well below the size of most or all of the bars in a sample, even for the most distant galaxies - as is the case for the distance-limited subsamples of $\mathrm{S}^{4} \mathrm{G}$ - then very few bars will be missed. But most SDSS-based studies have a typical linear resolution for their galaxies that is about an order of magnitude worse than that of $\mathrm{S}^{4} \mathrm{G}$. They will accordingly be much more vulnerable to resolution effects. And since the resolvability of bars depends on both survey resolution and their linear sizes, bars in lower-mass galaxies will be more easily missed than those in high-mass galaxies.

Figure 7 shows how the different resolutions interact with the distribution of bar sizes by plotting $\mathrm{S}^{4} \mathrm{G}$ observed bar size $a_{\text {vis }}$ versus stellar mass, $g-r$ colour, and $f_{\text {gas }}$. (Using the observed sizes helps incorporate the effects of inclination and projection.) The typical linear resolutions of both $\mathrm{S}^{4} \mathrm{G}$ (165 pc for Sample 1's mean distance of $17 \mathrm{Mpc}$, assuming $\left.\mathrm{FWHM} \approx 2^{\prime \prime}\right)$ and GZ2 (1.25 kpc for $\langle z\rangle=0.045$, assuming $\left.\mathrm{FWHM}=1.4^{\prime \prime}\right)$ are indicated on the figure with horizontal black (for $\mathrm{S}^{4} \mathrm{G}$ ) and blue (for GZ2) lines. ("GZ2" should be understood as representing typical SDSS-based bar surveys, not just the GZ2 studies.) Since Figure 3 suggests that a plausible lower limit for bar detectability is $a \sim 2 \times$ FWHM, I also plot twice the mean FWHM values for each case, using thinner dashed lines. The mean $\mathrm{S}^{4} \mathrm{G}$ resolution is clearly well below the observed sizes of almost all the bars. But the mean SDSS resolution excludes a significant fraction of the bars, and does so in a clearly differential fashion: more bars are below the resolution limit for lower stellar masses, for bluer colours, and for higher $f_{\text {gas }}$ values. This means that SDSSbased studies are likely to detect fewer of the bars in lowermass, blue, and gas-rich galaxies. (The implied trend is less clear for bar size versus galaxy colour, with bars apparently becoming larger in the bluest galaxies. The problem is that these are the galaxies for which local colour information is most incomplete.)

\subsubsection{Evidence for Missing Small Bars in SDSS Studies}

Is there more direct evidence that large-scale SDSS-based surveys have preferentially missed smaller bars? The left panel of Figure 8 compares the observed sizes of bars as a function of stellar mass from GZ2, using the published bar sizes of Hoyle et al. (2011) and stellar masses estimated (using the colour-to- $M / L$ relations of Zibetti et al. 2009) from $i$-band absolute magnitudes and $g-i$ colours in the GZ2 catalogs (Willett et al. 2013). The solid blue line is a LOESS (locally weighted regression) fit, somewhat analogous to a running mean (using the implementation of Cappellari et al. 2013); the dashed lines outline the $90 \%$ quantile limits, using the LOESS-based approach of Sakov et al. (2010). The distribution of $\mathrm{S}^{4} \mathrm{G}$ bar sizes is shown in the right panel of the same figure, along with corresponding LOESS fit and $90 \%$ quantiles in red.

By comparing the overall trends (red lines versus blue lines), we can see that while the GZ2 and $S^{4} \mathrm{G}$ bars span a similar range of sizes at the high-mass end (i.e., $\left.\log \left(M_{\star} / M_{\odot}\right) \sim 11\right)$, the distributions separate as one goes to lower masses, with the GZ2 distribution biased to larger sizes relative to the $\mathrm{S}^{4} \mathrm{G}$ bars. By the time we reach $\log \left(M_{\star} / M_{\odot}\right) \sim 10.2$, almost $90 \%$ of the GZ2 bars are larger than the typical $\mathrm{S}^{4} \mathrm{G}$ bar.

The distributions of $\mathrm{GZ2}$ and $\mathrm{S}^{4} \mathrm{G}$ bar sizes appear to converge again for much lower stellar masses (e.g., $\left.\log \left(M_{\star} / M_{\odot}\right) \lesssim 9\right)$, though the lower boundary for $\mathrm{S}^{4} \mathrm{G}$ sizes remains clearly below that for the GZ2 galaxies. This behavior might reflect the increase in relative bar size (bar semi-major axis divided by galaxy optical radius) at lower masses, as shown in Figure 9.

\section{SIMULATING SDSS BAR OBSERVATIONS}

As shown in the previous section, the distribution of bar sizes in the local universe suggests that a significant number of bars could be missed in SDSS-based surveys, for the simple reason that they are too small in angular size to be reliably detected. From Figure 7, we can deduce that SDSS-based studies will preferentially miss bars in lower-mass, bluer, and more gas-rich galaxies, because those are the galaxies with smaller bars; Figure 8 suggests that detected bars in GZ2 

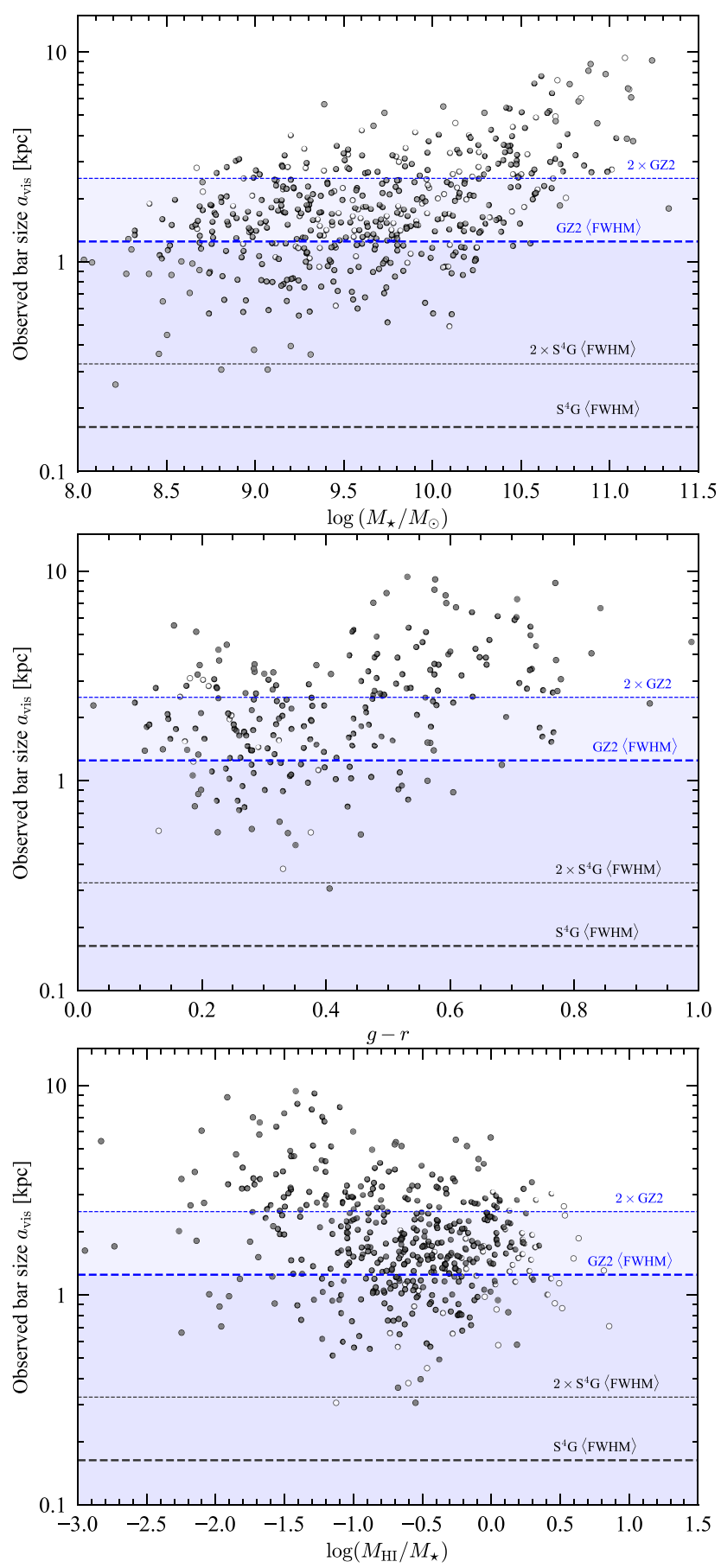

Figure 7. Observed (not deprojected) sizes of bars in $\mathrm{S}^{4} \mathrm{G}$ galaxies versus stellar mass (top), $g-r$ colour (middle), and gas mass ratio (bottom). Filled circles indicate galaxies in Sample $1(D \leqslant$ $25 \mathrm{Mpc}$, top panel) or Sample $1 \mathrm{~m}\left(D \leqslant 25 \mathrm{Mpc}, \log \left(M_{\star} / M_{\odot}\right) \geqslant\right.$ 8.5 , middle and bottom panels); open circles show additional galaxies with $D=25-30 \mathrm{Mpc}$ and also $\log \left(M_{\star} / M_{\odot}\right) \geqslant 9$ (middle and bottom panels). Horizontal dashed lines show typical linear resolution for galaxies in $\mathrm{S}^{4} \mathrm{G}$ (black, FWHM $=2^{\prime \prime},\langle D\rangle=17 \mathrm{Mpc}$ for Sample 1) and in GZ2 or similar SDSS-based studies (blue, $\left.\mathrm{FWHM}=1.4^{\prime \prime},\langle z\rangle \approx 0.045\right)$. Even assuming that all bars with angular sizes down to one FWHM are detected, SDSS-based surveys will miss a significant fraction of bars in lower-mass, blue, and gas-rich galaxies (dark shaded regions). More bars will be missed if the actual detection limit is closer to $2 \times \mathrm{FWHM}$ (dark + light shaded regions). studies are indeed biased toward larger sizes relative to local bars, and that this bias increases toward lower galaxy masses.

Can we test this idea more rigorously? The $\mathrm{S}^{4} \mathrm{G}$-based subsamples I use are defined to be volume- and mass-limited, which makes them potentially a good match to many of the SDSS-based samples. ${ }^{8}$ This means that it is possible to use the $S^{4} G$ galaxies - and their measured bar sizes - as a parent sample for simulating how local galaxies would be classified if they were observed with typical SDSS resolution at typical SDSS redshifts. Since the local subsamples are $\sim 97 \%$ complete in terms of gas mass fraction, they are also suitable for examining simulated bar fractions as a function of $f_{\text {gas }}$ (although the incompleteness of the actual SDSS studies in terms of $f_{\text {gas }}$ makes comparison more difficult).

The basic idea is simple: a mock sample of $N$ galaxies (where $N$ is similar in size to typical SDSS samples) is generated by sampling with replacement from an $\mathrm{S}^{4} \mathrm{G}$-based subsample and assigning each galaxy a random redshift drawn from the mock sample's redshift range, using uniform volume weighting. Since the relevant SDSS-based studies generally only have galaxies with $\log \left(M_{\star} / M_{\odot}\right) \gtrsim 9-9.5$ (e.g., Barazza et al. 2008; Masters et al. 2012; Cheung et al. 2013; Oh et al. 2012; Cervantes Sodi 2017), the best $\mathrm{S}^{4} \mathrm{G}$-based subsample to use is probably Sample $2 \mathrm{~m}: \log \left(M_{\star} / M_{\odot}\right) \geqslant 9$, $D \leqslant 30 \mathrm{Mpc}$. (For the gas-mass-ratio comparison, I limit the starting simulation sample to $\log \left(M_{\star} / M_{\odot}\right) \geqslant 9.5$.) If the original $\mathrm{S}^{4} \mathrm{G}$ galaxy is unbarred, then the sampled galaxy is assumed to be correctly identified as such. If the original galaxy does have a bar, then the orientation of the sampled galaxy's bar within its disc is chosen randomly from $\left(0,90^{\circ}\right)$ relative to the line of nodes, the galaxy inclination $i$ is chosen randomly from $\left(0,60^{\circ}\right)$, weighted by $\cos i$, and the observed bar size is computed by projecting the intrinsic $\mathrm{S}^{4} \mathrm{G}$ bar semi-major axis according to the chosen orientation. The galaxy is then "observed" to determine if its bar is detected, with the projected bar size in $\mathrm{kpc}$ converted to an angular size given the assigned redshift. If this is larger than the assumed angular-size limit of $2 \times \mathrm{FWHM}$, then the galaxy is classified as "barred"; otherwise, it is classified as "unbarred". The detected bar frequencies are then calculated for each stellar-mass or gas-mass-fraction bin. The process is repeated 200 times, with the median and $68 \%$ confidence intervals calculated from the results.

A sharp cutoff based solely on bar size, such as I simulate here, is obviously not terribly realistic. Bar strength (however defined) is probably important at some level, despite the evidence that the SDSS-based studies are not preferentially selecting strong bars (Section 3.4): bars which are, for example, especially elliptical and high-contrast may still be detectable even if their projected size is slightly below the resolution limit, while rounder, lower-contrast bars may be missed even if their projected size is slightly above the limit, even though both will be strongly affected by convolution with the seeing. Another complication is that the actual seeing for the SDSS images is variable; some images

8 Somewhat less so at lower masses, as the SDSS-based studies are really absolute-magnitude-limited, so that stellar-mass incompleteness starts to become a problem for $\log \left(M_{\star} / M_{\odot}\right)<10$ or so. 

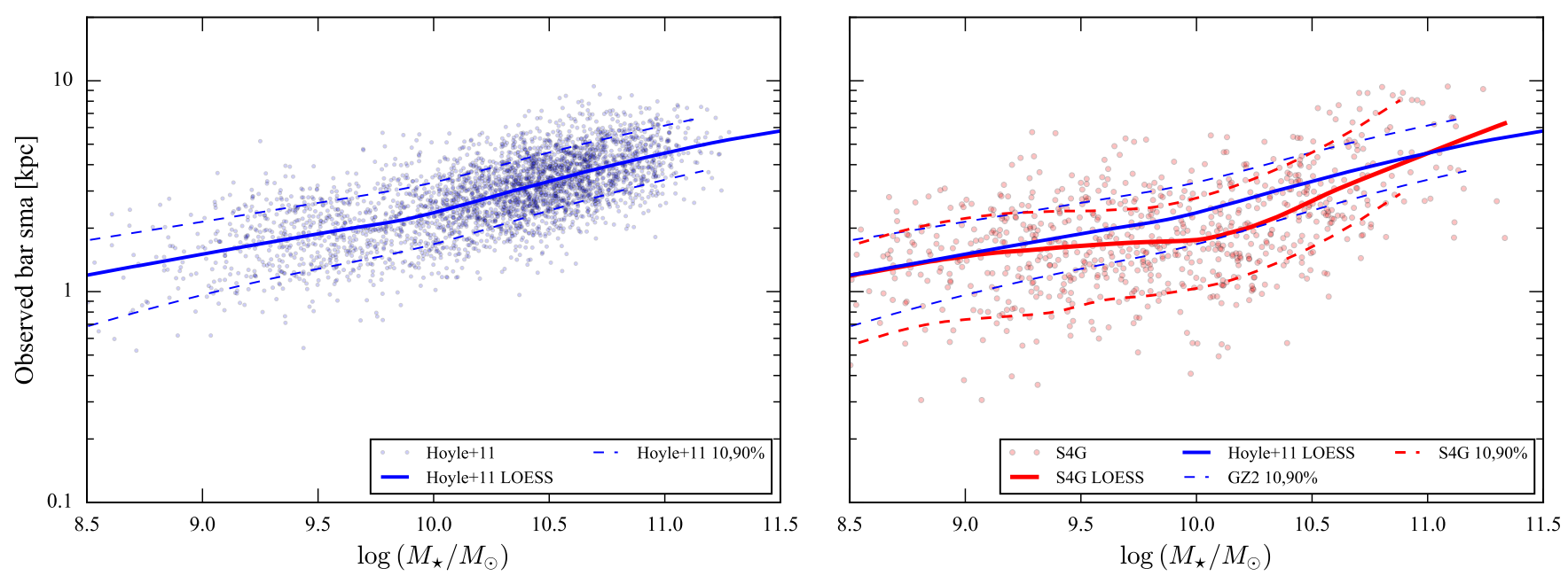

Figure 8. Comparison of observed bar sizes between GZ2 and $\mathrm{S}^{4} \mathrm{G}$. Left: Observed GZ2 bar sizes from Hoyle et al. (2011), as a function of stellar mass. The solid blue line is a LOESS fit to the data; the dashed lines outline the 10-90\% quantile range. Right: Same, but now showing $S^{4} \mathrm{G}$ observed bar sizes, with red lines indicating LOESS and 10-90\% quantiles. The blue lines repeat the GZ2 LOESS and quantile ranges from the left panel.

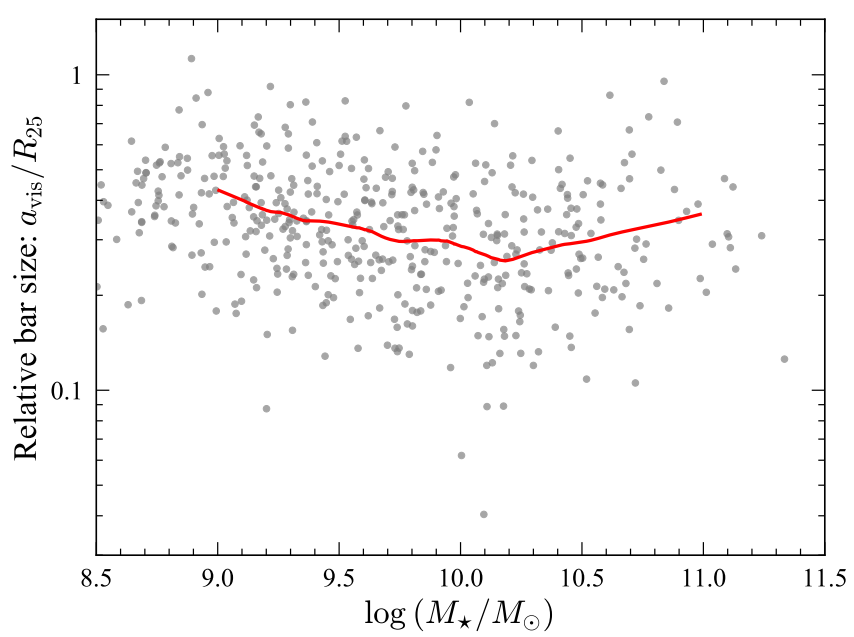

Figure 9. Relative bar size (bar semi-major axis divided by blue optical radius $R_{25}$ ) for $\mathrm{S}^{4} \mathrm{G}$ galaxies in Sample 2. The red line indicates a LOESS fit to galaxies with $\log \left(M_{\star} / M_{\odot}\right)=9-11$. Note that relative bar size reaches a minimum at $\log \left(M_{\star} / M_{\odot}\right) \sim 10.2$, and is actually largest for stellar masses of $\log \left(M_{\star} / M_{\odot}\right) \sim 9$. See also Fig. 20 of Díaz-García et al. (2016a), which shows a similar trend for bar size relative to near-IR galaxy size as a function of stellar mass.

may have unusually good seeing, making smaller bars more detectable, while other images with worse-than-typical seeing will make detecting larger bars more difficult. A further potential issue is that the $\mathrm{S}^{4} \mathrm{G}$ sample - despite containing galaxies from the Virgo and Fornax Clusters - does not have any galaxies from very massive clusters, and so the environmental match to the larger, SDSS-based samples is not perfect. Finally, the $\mathrm{S}^{4} \mathrm{G}$ subsample I use excludes $\mathrm{S} 0$ galaxies, although some of the SDSS-based studies exclude "early-type" galaxies themselves.

These issues aside, I believe it is still useful to investigate how well the SDSS-based bar-fraction trends can be reproduced - even if only qualitatively - by this simple resolution-effects model. The next two subsections explore this for the cases of stellar mass and gas mass ratio.

\subsection{Simulated Bar Fraction and Stellar Mass}

The left-hand panel of Figure 10 shows the median trend for $f_{\text {bar }}$ versus $\log \left(M_{\star} / M_{\odot}\right)$ from 200 bootstrapped mock samples, each with $N=10,000$ galaxies. The basic trend seen in GZ2 (Masters et al. 2012; Melvin et al. 2014) and at least some other SDSS-based studies (e.g., Oh et al. 2012; Gavazzi et al. 2015; Cervantes Sodi 2017) is clearly reproduced: $f_{\text {bar }}$ is very low for galaxies with $\log \left(M_{\star} / M_{\odot}\right)<10$, and then increases steeply for higher masses.

The relation seen by Nair \& Abraham (2010b) is not as well reproduced - specifically, the increase in $f_{\text {bar }}$ to lower stellar masses $\left(\log \left(M_{\star} / M_{\odot}\right)<10\right)$ that Nair \& Abraham reported is not apparent, although GZ2 and other SDSS studies did not report much of a trend in this sense, either. In this case, difference in how bar detection works in the different studies may be important. In particular, it could be that the Nair \& Abraham study is more sensitive to both the relative size of bars and the absence of bulges in low-mass galaxies. The first factor is illustrated by Figure 9 (see also Fig. 20 of Díaz-García et al. 2016a), which shows that bar size relative to optical disc size actually increases to lower stellar masses for $\log \left(M_{\star} / M_{\odot}\right) \lesssim 10$. It may thus be easier in some cases to identify bars in lower-mass galaxies simply because they span a larger part of the visible disc. The minimum in relative bar size happens at $\log \left(M_{\star} / M_{\odot}\right) \approx 10.2-$ which is where the Nair \& Abraham (2010b) bar frequency has its minimum. This might also explain why Cervantes 

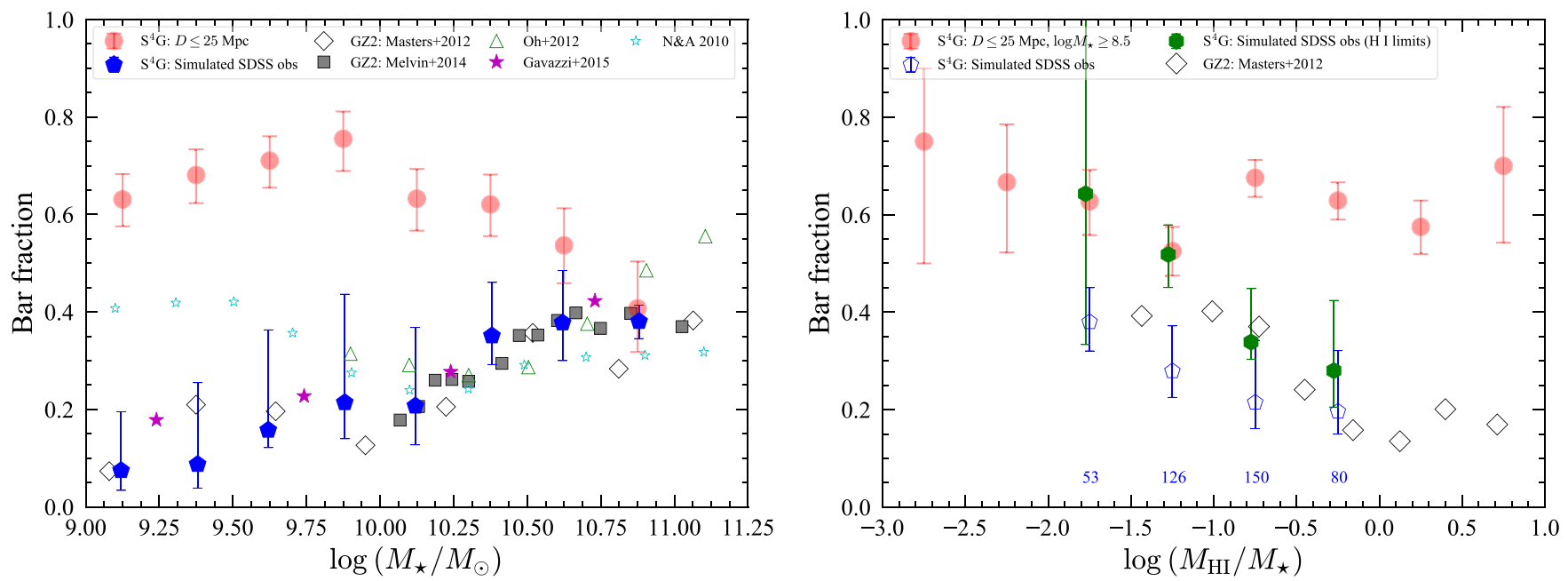

Figure 10. Observed and simulated bar frequencies as a function of stellar mass and $f_{\text {gas }}$. In both panels, observed bar fractions for $\mathrm{S}^{4} \mathrm{G}$ galaxies are shown with filled red circles (see Figure 5). Left: bar fractions as function of stellar mass, with SDSS-based fractions from Masters et al. (2012) and Melvin et al. (2014) in grey, from Oh et al. (2012) with open green triangles, from Gavazzi et al. (2015) with filled magenta stars, and from Nair \& Abraham (2010b) with open cyan stars. Filled blue pentagons show median bar fractions (with $68 \%$ confidence intervals) for simulated observations of $\mathrm{S}^{4} \mathrm{G}$ galaxies at typical SDSS-survey redshifts $(z=0.01-0.05)$, assuming that bars with (projected) semi-major axes smaller than twice the typical FWHM of SDSS images are not detected. Right: same, but now showing bar fractions as function of $f_{\text {gas }}$, including GZ2 fractions from Masters et al. (2012). Open blue pentagons are from simulations similar to those in the left panel; green hexagons - slightly offset for clarity - are from simulations that include a simplistic H I selection function based on Haynes et al. (2011). The blue numbers along the bottom of the plot are the number of $\mathrm{S}^{4} \mathrm{G}$ galaxies in each bin in the $\log \left(M_{\star} / M_{\odot}\right) \geqslant 9.5$ parent sample used for the simulation.

Sodi (2017) reports a sharp upturn in $f_{\text {bar }}$ for his very lowestmass bin (his Fig. 1), along with the weak upturns in $f_{\text {bar }}$ for very blue and low-mass galaxies in GZ2 (e.g., Masters et al. 2011, 2012).

A second possible reason for the high $f_{\text {bar }}$ values seen for low masses (and blue galaxies) by Nair \& Abraham (2010b) is that their sample is magnitude-limited $(g<16)$, unlike e.g. the GZ2 studies, and unlike the volume-limited subsamples of $\mathrm{S}^{4} \mathrm{G}$ constructed for this paper. A magnitude limit means that less luminous galaxies (which tend to be lower in mass and bluer) are preferentially found at smaller redshifts, as can be seen in Fig. 20 of Nair \& Abraham (2010a): for example, galaxies with $\log \left(M_{\star} / M_{\odot}\right)=8.0-9.5$ are mostly found at $z \approx 0.01-0.02$, while galaxies with $\log \left(M_{\star} / M_{\odot}\right)=10.8-$ 11.2 are mostly found at $z \approx 0.04-0.06$. This means that a typical low-mass galaxy will be observed with several times better spatial resolution than a typical high-mass galaxy in their sample. It is conceivable that the upturn in $f_{\text {bar }}$ toward lower stellar masses in Fig. 1 of Nair \& Abraham (2010b) is at least partly due to this effect - especially since the $\mathrm{S}^{4} \mathrm{G}$ samples show that galaxies with $\log \left(M_{\star} / M_{\odot}\right) \lesssim 10.1$ have bar sizes that depend rather weakly on stellar mass (Figure 6).

Unfortunately, I cannot properly test this last hypothesis using the $\mathrm{S}^{4} \mathrm{G}$ sample, because the $g=16$ limit of Nair \& Abraham (2010a) is almost a full magnitude fainter than the $\mathrm{S}^{4} \mathrm{G}$ parent-sample magnitude limit of $B=15.5$. This means that the Nair \& Abraham sample has many galaxies fainter than are found in $\mathrm{S}^{4} \mathrm{G}$, making it difficult to compare the samples.

Leaving aside the caveats regarding the Nair \& Abraham (2010b) study, I conclude that the interaction of the bar-size-stellar-mass correlation and low spatial resolution explains most, if not all, of the apparent correlation between $f_{\text {bar }}$ and stellar mass reported by the majority of SDSSbased studies.

\subsection{Simulated Bar Fraction and Gas Mass Ratio}

The right-hand panel of Figure 10 shows simulated SDSS observations of $f_{\text {bar }}$ as a function of $f_{\text {gas }}$. Results from simulations using the same approach as for the $\log \left(M_{\star} / M_{\odot}\right)$ comparison - except now using a sample size of 2000 as a better match to that used by Masters et al. (2012), and with stellar masses restricted to $\log \left(M_{\star} / M_{\odot}\right) \geqslant 9.5$ - are shown using hollow blue pentagons. As expected, the observed bar fraction decreases to higher gas mass ratios. This qualitatively agrees with the results of Masters et al. (2012) and Cervantes Sodi (2017). The quantitative match is not as good as the agreement between the simulated and reported $f_{\text {bar }}{ }^{-}$ $\log \left(M_{\star} / M_{\odot}\right)$ results (left-hand panel of the figure). This is at least partly because the SDSS $f_{\text {gas }}$ results include complicated selection effects based on the varying detection efficiency of the ALFALFA survey as a function of gas content and redshift (see the discussion in Masters et al. 2012).

Inspection of Fig. 2 of Masters et al. (2012) suggests that two related biases may be relevant. Most of their gasrich galaxies (e.g., $\log f_{\text {gas }}=0-0.5$ ) are low-mass - and thus have smaller intrinsic bar sizes - and are mostly at higher redshifts (the majority between $z \sim 0.03$ and 0.05 ). In contrast, most of their gas-poor galaxies (e.g., $\log f_{\text {gas }}=-1.5$ to -1.0) are high-mass - and thus have larger intrinsic bar sizes - and at the same time are at lower redshifts (mostly between $z \sim 0.015$ and 0.035 ). This is of course a natural result for the combination of a flux-limited H I survey and the 
strong anticorrelation between gas content and stellar mass. So gas-rich galaxies in SDSS + ALFALFA samples will tend to have small intrinsic bar sizes and be further away, which will lower the bar-detection efficiency, while gas-poor galaxies will have intrinsically larger bars and be closer, which will increase the bar-detection efficiency. This effect is a plausible explanation for the fact that Masters et al. (2012) and Cervantes Sodi (2017) find $f_{\text {bar }}$ to be anticorrelated with gas mass ratio at fixed stellar mass (or, equivalently, $f_{\text {bar }}$ increases with $\mathrm{H}$ I deficiency, which is computed as a function of stellar mass).

As an attempt to include the effects of the $\mathrm{H}$ I detection bias of the SDSS studies, I generated additional simulated surveys using the suggested $50 \%$ detection limit for ALFALFA from Eqns. 6 and 7 of Haynes et al. (2011), with the HyperLeda vmaxg parameter standing in for $W_{50}$ (i.e., $W_{50}=2 \mathrm{vmaxg}$ ). For each potential $\mathrm{S}^{4} \mathrm{G}$ galaxy chosen for the bootstrapped sample, I computed its hypothetical H I flux (using the randomly chosen redshift and the galaxy's actual $\mathrm{H}$ I flux from HyperLeda) and the ALFALFA limiting flux. If the hypothetical flux at redshift $z$ was brighter than the limiting flux, then the galaxy was accepted into the bootstrapped sample; if its $\mathrm{H}$ I flux was too faint, it was rejected and a new galaxy selected from the parent sample, with the process repeated until a galaxy was accepted. The results of this "H I-limited" simulation are shown using green hexagons in the right panel of Figure 10. These observed bar fractions are higher than for the standard simulation (hollow blue pentagons), because the $\mathrm{H}$ I-selection cutoff tends to exclude more distant galaxies, where bars are harder to detect. The overall pattern of increasing bar fraction to lower $f_{\text {gas }}$ is even stronger, and the predicted $f_{\text {bar }}$ values tend to overlap somewhat better with the GZ2 values from Masters et al. (2012).

This suggests that we can explain the discrepancy between the absence of a dependence of bar fraction on gas fraction in $\mathrm{S}^{4} \mathrm{G}$ galaxies and the apparent strong dependence seen in SDSS studies, at least to first order, via a combination of the dependence of bar size on stellar mass - and thus indirectly on $f_{\text {gas }}-$ and the effects of strong $\mathrm{H}$ I flux limits.

\subsection{Bar Fraction and Colour}

Masters et al. (2011, 2012) and Lee et al. (2012a) reported strong trends between bar fraction and galaxy optical colour, in the sense that redder galaxies are much more likely to be barred. As noted in Section 3.2, the local $\left(\mathrm{S}^{4} \mathrm{G}\right)$ galaxies do not show any evidence for this; if anything, the local trend suggests a very slight (though statistically not significant) decrease in $f_{\text {bar }}$ for redder galaxies; a similar trend was seen by Barazza et al. (2008).

Unfortunately, the incompleteness of colour data for $\mathrm{S}^{4} \mathrm{G}$ galaxies (see Appendix A) makes it difficult to construct simulated SDSS observations as I have done for the stellarmass and $f_{\text {gas }}$ cases, particularly since the incompleteness is worse for bluer galaxies.

Nonetheless, given that galaxy colour does correlate with stellar mass, it is very likely that the reported $f_{\mathrm{bar}}-$ colour correlations from SDSS-based studies are mostly if not entirely side effects of differential bar detection: since bluer galaxies tend to be lower mass, they also tend to have smaller bars, which will be harder to detect (middle panel of Figure 7). The fact that the mean resolution of Barazza et al. (2008) was about twice as good as that of the GZ2 and Lee et al. (2012a) studies, combined with a possible higher bar-detection efficiency, likely explains why the Barazza et al. colour trend is similar to the $\mathrm{S}^{4} \mathrm{G}$ trend.

\section{DISCUSSION}

\subsection{The Primacy of Stellar Mass}

Section 3.1 suggests that bar presence depends quite strongly on stellar mass, with little or no dependence on galaxy colour or (neutral) gas content. We can still ask whether - once we account for the main stellar-mass dependence - there might not still be some residual dependences of bar frequency on colour or gas content. Since the quadratic logistic regression is such a good match to the bar frequency as a function of stellar mass, I test for possible residual correlations by performing logistic regressions using both stellar mass and one or both of the other parameters as independent variables. This is in essence a variation of Equation 6, with the bar frequency described as

$f_{\mathrm{bar}}(x)=\frac{1}{1+e^{-\left(\alpha+\beta_{1} m+\beta_{2} m^{2}+\beta_{3} x\right)}}$,

where $m=\log \left(M_{\star} / M_{\odot}\right)$ and $x=$ either $g-r$ or $\log f_{\text {gas }}$.

Table 4 shows the resulting best-fit parameters for the fit using stellar mass and colour and the fit using stellar mass and $f_{\text {gas }}$. Each fit is done to two subsets of data: galaxies in Sample $1 \mathrm{~m}$ with $f_{\text {gas }}$ values (556 galaxies) and galaxies in Sample $1 \mathrm{~m}$ with $g-r$ values (319 galaxies). Akaike Information Criteria (AIC) values from the fits can be used to evaluate the relative goodness of different fits, but only within the individual subsamples. In both subsamples, the lowest AIC values - and thus the preferred fits - are for the quadratic dependence on $\log \left(M_{\star} / M_{\odot}\right)$ alone. Adding either gas mass ratio or colour to the fits results in higher AIC values - significantly so $(\Delta \mathrm{AIC}=6.6)$ for the large, $f_{\text {gas }}$-based sample. This is a reasonably clear indication that the bar frequency does not show any significant dependence on either gas mass ratio or $g-r$ colour, once the strong dependence on stellar mass is accounted for.

\subsection{Implications for High-Redshift Bar Studies}

Could differential resolution affect high- $z$ bar studies? Studies using HST data of galaxies at moderate redshifts (e.g. $z \sim 0.4-1.0$ ) sometimes make the same sort of claims that they are incapable of missing "normal" bars that some SDSSbased studies have made. Whether this is true depends partly on resolution and partly on what the (unknown) distribution of bar sizes at different redshifts actually is. The spatial resolution of $H S T$ images for moderate-redshift galaxies is better than typical $z \sim 0.05$ SDSS images: for example, even at $z=1$, an $I$-band $H S T$ image will have a FWHM of $\sim 0.8 \mathrm{kpc}$, compared with $1.25 \mathrm{kpc}$ for a typical SDSS image at $z=0.05$. Clearly, fewer bars should be missed by HST surveys - if bars have the same size distribution at high redshift as they do locally.

Figure 11 compares potential high-redshift resolution limits with the $S^{4} \mathrm{G}$ bar-size distribution: I show linear 
Table 4. Logistic Regression Results: Multiple Variables

\begin{tabular}{|c|c|c|c|c|}
\hline $\begin{array}{l}\text { Variable } \\
\text { (1) }\end{array}$ & $\begin{array}{r}\alpha \\
(2)\end{array}$ & $\begin{array}{r}\beta \\
(3)\end{array}$ & $\begin{array}{l}P_{\beta} \\
(4)\end{array}$ & $\begin{array}{r}\mathrm{AIC} \\
(5)\end{array}$ \\
\hline \multicolumn{5}{|c|}{ Subsample: 556 galaxies with $f_{\text {gas }}$ values } \\
\hline $\log M_{\star}$ & -6.05 & 0.69 & 0.0012 & 799.6 \\
\hline $\begin{array}{l}\log M_{\star} \\
\left(\log M_{\star}\right)^{2}\end{array}$ & $\begin{array}{r}-84.29 \\
-\end{array}$ & $\begin{array}{r}17.55 \\
-0.90\end{array}$ & $\begin{array}{l}0.00016 \\
0.00019\end{array}$ & $\begin{array}{r}762.1 \\
-\end{array}$ \\
\hline $\begin{array}{l}\log M_{\star} \\
\left(\log M_{\star}\right)^{2} \\
\log f_{\text {gas }}\end{array}$ & $\begin{array}{r}-84.51 \\
-\end{array}$ & $\begin{array}{r}17.51 \\
-0.90 \\
0.21\end{array}$ & $\begin{array}{r}0.00020 \\
0.00024 \\
0.55\end{array}$ & $\begin{array}{r}768.7 \\
- \\
-\end{array}$ \\
\hline \multicolumn{5}{|c|}{ Subsample: 319 galaxies with $g-r$ values } \\
\hline $\log M_{\star}$ & -6.05 & 0.69 & 0.0012 & 439.2 \\
\hline $\begin{array}{l}\log M_{\star} \\
\left(\log M_{\star}\right)^{2}\end{array}$ & $\begin{array}{r}-75.16 \\
-\end{array}$ & $\begin{array}{r}15.35 \\
-0.78\end{array}$ & $\begin{array}{l}0.037 \\
0.037\end{array}$ & $\begin{array}{r}433.5 \\
-\end{array}$ \\
\hline $\begin{array}{l}\log M_{\star} \\
\left(\log M_{\star}\right)^{2} \\
g-r\end{array}$ & $\begin{array}{r}-67.25 \\
- \\
-\end{array}$ & $\begin{array}{r}13.62 \\
-0.68 \\
-1.47\end{array}$ & $\begin{array}{r}0.071 \\
0.077 \\
0.21\end{array}$ & $\begin{array}{r}434.5 \\
- \\
-\end{array}$ \\
\hline
\end{tabular}

Results of multivariable logistic regression for bar frequency $f_{\text {bar }}$ in Sample $1 \mathrm{~m}\left(\mathrm{~S}^{4} \mathrm{G}\right.$ spirals with $D \leqslant 25 \mathrm{Mpc}$ and $\log \left(M_{\star} / M_{\odot}\right)>$ $8.5)$ as a function of log of stellar mass and either $g-r$ colour or gas mass fraction $f_{\text {gas }}$. Two subsamples are considered: the first is all galaxies in Sample $1 \mathrm{~m}$ with $f_{\text {gas }}$ values, while the second is all galaxies with $g-r$ colours. (1) Galaxy parameter used in fit. (2) Intercept value for fit. (3) Slope for fit. (4) $P$-value for slope. (5) Akaike Information Criterion value for fit; values should be compared only within a given subsample, with lower values indicating better fits.

FWHM for two different redshifts $(z=0.4$ and 1.0, bracketing the range usually used in $H S T$ bar studies), assuming an I-band angular PSF FWHM of $0.09^{\prime \prime}$ (e.g., for F814W or F850LP filters used with ACS-WFC or WFC3-UVIS). If bars can be reliably identified in $H S T$ images down to $a \sim$ FWHM, then relatively few bars should be missed, at least for $\log \left(M_{\star} / M_{\odot}\right) \gtrsim 10$. But if a more conservative limit of $2 \times$ FWHM is used - as would seem wise for GZ2-style studies such as Melvin et al. (2014) and Simmons et al. (2014) then we might expect lower bar fractions at higher redshifts purely from resolution effects, and this could apply to stellar masses as high as $\sim 10^{10.5} M_{\odot}$.

There are actually two things to worry about in terms of resolution for $H S T$ bar studies. The first is the high-redshift equivalent of what happens for the low-redshift SDSS studies: the fact that a resolution limit near or above the size of the smallest bars, combined with a dependence of bar size on galaxy mass, can create - or exaggerate - a trend of $f_{\text {bar }}$ with stellar mass. The second is that comparing bar fractions at different redshifts means comparing different resolutions, for the simple reason that higher-redshfit samples are observed with lower spatial resolution. As Figure 11 shows, going from $z=0.4$ to $z=1.0$ will lower the detected bar fraction, creating an apparent evolution in the $f_{\text {bar }}$ with redshift qualitatively similar to what has actually been reported.

One way to address the second confounding factor the change in resolution with redshift - would be to convolve images of lower-redshift galaxies to the worst-case spatial resolution of the high-redshift limit of a survey, so that all galaxies are observed with the same spatial resolution.
Unfortunately, this seems to be something that is never attempted.

The preceding argument is based on the undoubtedly unrealistic assumption that bars at high redshift having the same distribution of sizes that they do in the local universe. Most theoretical studies and models suggest that bars should grow in size over time (e.g., Debattista \& Sellwood 2000; Martinez-Valpuesta et al. 2006; ?), generally by factors of $\sim 50-100 \%$. But this makes the resolution problem worse, because bars at higher redshifts would then be smaller and thus even harder to detect.

A simplistic demonstration of the possible effects is shown in Figure 12, which repeats the simulated observations approach of Section 5, but places all resampled $S^{4} G$ galaxies at three different redshift ranges, corresponding to the ranges used in the study of HST COSMOS data by Sheth et al. (2008), and observes them with a typical HST $I$-band FWHM of $0.09^{\prime \prime}$. Red diamonds show the results using the $S^{4} \mathrm{G}$ spiral sample as is, while the orange pentagons show the results when their bar sizes are set to half their actual values. The black stars are the observed $f_{\text {bar }}$ values from Sheth et al. (2008). ${ }^{9}$

If bars do not grow significantly in size, so that they were already as large at higher redshifts as they are now, then the simulations overpredict bar frequencies for all galaxies in the high redshift regime $(z=0.60-0.84$, top panel of Figure 12), and they overpredict bar frequencies for lowermass $\left(\log \left(M_{\star} / M_{\odot}\right) \lesssim 10.5\right)$ galaxies in the medium redshift regime $(z=0.037-0.60$, middle panel $))$. For the lowest redshift regime $(z=0.14-0.37$, bottom panel $)$, the observed frequencies are generally consistent with the local values and possibly more consistent with observed true local values than the prediction for that redshift bin; this might be an indication that the $2 \times$ FWHM detection limit I assume underestimate the bar-detection efficiency of the methods used in Sheth et al. (2008).

If, on the other hand, bars at higher redshifts were only half their present size, then the simulations are a surprisingly good match for the high-redshift sample. In particular, the trend of increasing bar fraction for increasing stellar mass is fully reproduced, and the predicted frequencies are only marginally higher than the observed HST frequencies. For the intermediate redshifts, the simulations with smaller bar sizes slightly underpredict the observed frequencies, and they clearly underpredict the observed frequencies in the lowestredshift bin (bottom panel); this suggests that at those redshifts bars were already more than half their present size.

Taken together, these result suggest that a combination of significant growth in bar size and redshift-dependent resolution effects can in principle explain most, though perhaps not all, of the observed changes in bar frequency reported by Sheth et al. (2008). In particular, the simulations do a good job of reproducing the trends in bar frequency as a function of stellar mass - i.e., increasing with stellar mass over the range of $\log \left(M_{\star} / M_{\odot}\right) \sim 10.2-11$ - which are similar to those seen in most SDSS-based samples, and which differ so dramatically from the $\mathrm{S}^{4} \mathrm{G}$ trend (Figure 5).

9 These points are plotted at the center of their respective bins, in contrast to the original plot in Fig. 3 of Sheth et al. (2008), where they were plotted at the left side of their bins. 


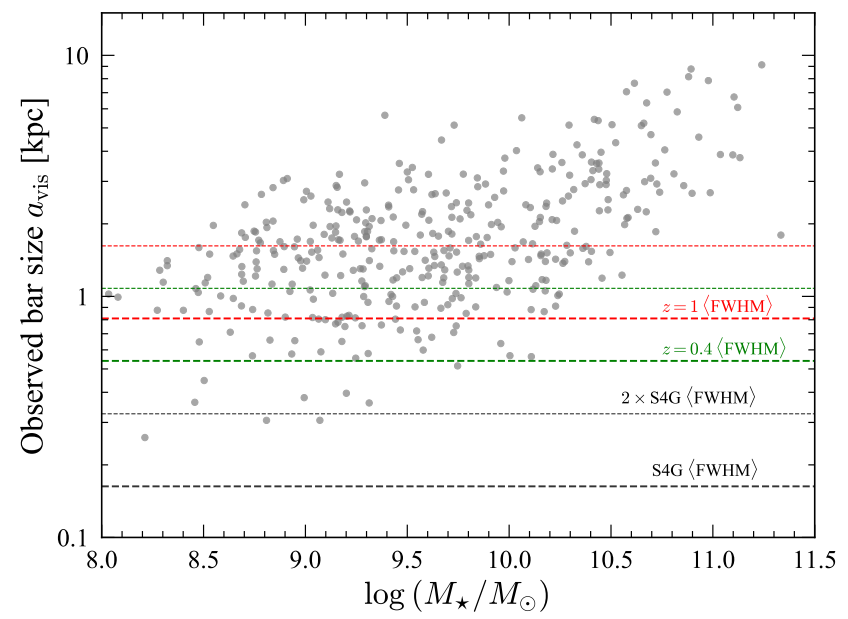

Figure 11. As for Figure 7 (upper panel), but now showing potential resolution limits at intermediate to high redshifts. Assuming a typical F814W FWHM of $\sim 0.09^{\prime \prime}$, the expected resolution limits (FWHM) at $z=0.4$ and 1.0 are shown with thick dashed green and red lines, respectively. Thin dashed lines show the $2 \times$ FWHM limit for each case.

A model where all bars were already in place at $z \sim 0.8$ but had only half their size, so that the observed redshift evolution is not due to any actual changes in bar frequency, is probably not realistic. The truth is almost certainly somewhere in between: that is, the apparent evolution in bar fraction with redshift reported by HST studies is probably real, but has likely been exaggerated by the combination of resolution effects and changes in bar sizes over cosmic time. Before we can say for certain how the bar fraction evolves with redshift - and with stellar mass - we need to be able to disentangle true evolution in bar frequencies from the evolution of resolution effects, and the evolution of bar sizes.

\subsection{Implications for Models of Bar Formation and Growth}

Theoretical studies of bar formation and evolution have suggested that bar formation in gas-rich discs is suppressed or at least delayed, and that once a bar forms its growth can also be slowed when the disc is gas-rich (e.g., Berentzen et al. 1998; Debattista et al. 2006; Athanassoula et al. 2013). Alternately, too much gas accretion might weaken or even destroy pre-existing bars (Bournaud \& Combes 2002; Bournaud et al. 2005). The SDSS-based results of Masters et al. (2012), Cheung et al. (2013), and Cervantes Sodi (2017) have been interpreted to support at least some of this, since they found lower bar fractions in galaxies with more gas (or in galaxies with higher star-formation rates, which can be interpreted as a proxy for gas content).

However, the fact that local galaxies show an essentially constant bar fraction as a function of (atomic) gas mass fraction (bottom panels of Figure 5) runs counter to much of the preceding. It clearly disagrees with the predictions of Bournaud \& Combes (2002) and Bournaud et al. (2005) that high gas content should rapidly destroy bars, although
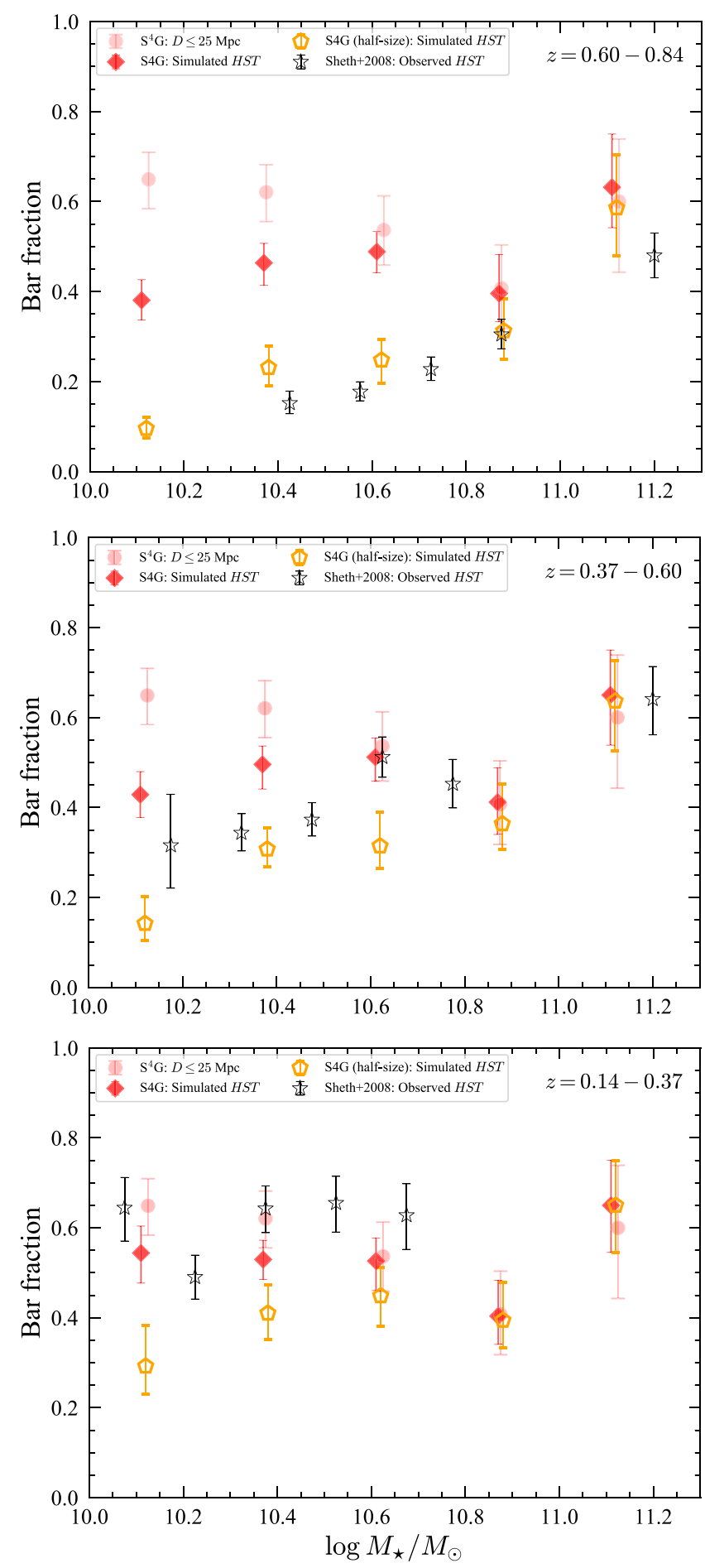

Figure 12. As for the left panel of Figure 10, but now showing simulated high-redshift observations with HST. Red circles show the local observed $\mathrm{S}^{4} \mathrm{G}$ bar fractions; red diamonds show simulated $H S T$ observations of $\mathrm{S}^{4} \mathrm{G}$ spirals assuming that bars with projected semi-major axes $<2 \times$ FWHM go undetected (using FWHM $=0.09^{\prime \prime}$ ), while orange pentagons show the same thing but also assuming that bar sizes were only half their present value. Black stars show observed HST total bar fractions from Sheth et al. (2008). Top: Simulations and data for $z=0.60-0.84$. Middle: $z=0.37-0.60$. Bottom: $z=0.14-0.37$. 
this is perhaps not surprising, as most recent simulations have found that bars are rarely if ever destroyed by high gas content (see., e.g., the discussion in Athanassoula et al. 2013). More generally, the fact that the majority of local spirals have $f_{\text {gas }} \gtrsim 0.1$ (Figure 2 ) and that the observed bar fraction is roughly constant from values of $f_{\text {gas }} \sim 0.01$ to 1 suggests that high gas content cannot be very effective in suppressing bar formation or growth over long time periods - unless any gas-driven delay is short enough that essentially all galaxies have already passed through it and formed their bars.

Only for very high gas mass fractions is there some evidence for an effect: as can be seen in the lower-right panel of Figure 5, the fraction of strong bars declines rather steeply for $f_{\text {gas }} \gtrsim 1$, while the fraction of weak bars goes up. This gas-mass-fraction level is higher than that explored in most bar simulations.

\section{SUMMARY}

In order to investigate how the local frequency of bars in spiral galaxies depends on galaxy mass and other properties, I constructed distance- and mass-limited subsamples of lowinclination $\left(i \leqslant 65^{\circ}\right)$ spiral galaxies from the Spitzer Survey of Stellar Structure in Galaxies $\left(S^{4} \mathrm{G}\right)$, using bar identifications and measurements from Herrera-Endoqui et al. (2015) and stellar masses from Muñoz-Mateos et al. (2015). I included colour and neutral-hydrogen measurements from HyperLeda and computed $V / V_{\max }$ weights to correct for the angular-diameter limit of $\mathrm{S}^{4} \mathrm{G}$; for the colours, I also computed $B_{\mathrm{tc}}$-based weights to correct for colour incompleteness in the HyperLeda data. The resulting subsamples include 851 galaxies with $D \leqslant 30 \mathrm{Mpc}$ and 659 with $D \leqslant 25$ $\mathrm{Mpc}$; the latter is the main subsample analyzed in this paper. Applying stellar-mass limits yields 638 galaxies with $\log \left(M_{\star} / M_{\odot}\right) \geqslant 9$ and $D \leqslant 30 \mathrm{Mpc}$, or 574 galaxies with $\log \left(M_{\star} / M_{\odot}\right) \geqslant 8.5$ and $D \leqslant 25$ Mpc.

The main findings of this analysis are the strong dependence of bar frequency on stellar mass and the absence of any clear dependence on galaxy colour or gas content. More specifically, the bar frequency in $\mathrm{S}^{4} \mathrm{G}$ galaxies peaks at $\log \left(M_{\star} / M_{\odot}\right) \approx 9.7$ with a value of $f_{\text {bar }}=0.70$ and declines to both lower and higher masses. On the other hand, $f_{\text {bar }}$ is roughly constant as a function of $g-r$ colour (or perhaps declining to redder colours, though this trend is not statistically significant) over the range $g-r \approx 0.1-0.8$, and is also roughly independent of $\mathrm{H}$ I gas mass fraction $\left(f_{\text {gas }}=M_{\mathrm{HI}} / M_{\star}\right)$.

These $f_{\text {bar }}$ trends differ quite strongly from most studies using large SDSS-based samples (typically at $z=0.01-0.5$ ), which tend to find that $f_{\mathrm{bar}}$ increases strongly toward higher masses (peaking at $\log \left(M_{\star} / M_{\odot}\right) \sim 11$ ), redder colours, and lower values of $f_{\text {gas }}$. The SDSS-based $f_{\text {bar }}$ values are almost always significantly lower than the $\mathrm{S}^{4} \mathrm{G}$ galaxies, except for the highest stellar masses and reddest colours.

I argue that this discrepancy between very local $\left(\mathrm{S}^{4} \mathrm{G}\right)$ and SDSS-based bar fractions can be explained primarily by a combination of two factors:

(i) Differences in effective spatial resolution due to differences in galaxy distances. Galaxies that are further away have bars that are harder to detect, given the finite angular resolution of images. The effective spatial resolution of $\mathrm{S}^{4} \mathrm{G}$ is $\sim 0.17 \mathrm{kpc}$ at the mean distance $(17 \mathrm{Mpc})$ of the $D \leqslant 25$ Mpc subsample, assuming an angular FWHM of $\approx 2^{\prime \prime}$. The equivalent for typical SDSS-based studies (mean redshift $\sim 0.045$, typical FWHM $\sim 1.4^{\prime \prime}$ ) is $\sim 1.3 \mathrm{kpc}$. A plausible angular-size limit for bar detection is $a_{\text {obs }} \sim 2 \times \mathrm{FWHM}$, which makes the mean linear-size limits $\sim 0.33 \mathrm{kpc}$ for $\mathrm{S}^{4} \mathrm{G}$ and $\sim 2.5 \mathrm{kpc}$ for typical SDSS-based surveys.

(ii) The dependence of bar size on galaxy stellar mass (and, less directly, on colour and gas content). Bar size is correlated with stellar mass (in a bimodal fashion, with a steeper relation for $\left.\log \left(M_{\star} / M_{\odot}\right) \gtrsim 10.2\right)$; bar sizes also exhibit significant scatter around the mean value for a given stellar mass.

The result is that bars in high-mass (red, gas-poor) galaxies are easier to find because they are larger. The effective resolution of the Spitzer images of $\mathrm{S}^{4} \mathrm{G} D \leqslant 25 \mathrm{Mpc}$ subsample is good enough so that almost all bars have observed semi-major axes - including projection effects - larger than $2 \times$ FWHM and can thus be detected, even for quite low stellar masses. But for distances typical of SDSS-based surveys, bar sizes start to drop below $2 \times$ FWHM for stellar masses $\log \left(M_{\star} / M_{\odot}\right) \lesssim 10.7$. The dispersion of bar sizes means that significant numbers of bars can be missed even though the mean bar size might still be above the resolution limit.

Comparison of the observed sizes of bars from GZ2, as tabulated by Hoyle et al. (2011), with sizes for $\mathrm{S}^{4} \mathrm{G}$ galaxies supports this general argument. GZ2 and $\mathrm{S}^{4} \mathrm{G}$ bars have similar sizes at very high masses $\left(\log \left(M_{\star} / M_{\odot}\right) \sim 11\right)$; but as one goes to lower masses, the average for $\mathrm{S}^{4} \mathrm{G}$ bars falls below that of GZ2 bars, until by $\log \left(M_{\star} / M_{\odot}\right) \sim 10.2$, the average $\mathrm{S}^{4} \mathrm{G}$ bar is smaller than $\sim 90 \%$ of GZ2 bars. This is a clear indication that GZ2 is systematically missing smaller bars at lower masses, which will mean a lower apparent bar fraction. (The bars that are being missed are not a separate population of "nuclear" bars, but simply the lower end of the distribution of normal galactic bars.)

An additional bias applies to SDSS-based studies using $f_{\text {gas }}$ (Masters et al. 2012; Cervantes Sodi 2017), because only galaxies with enough gas to be detected by ALFALFA were included in the samples. This means that galaxies with high gas mass fractions tended to be observed out to larger distances than galaxies with low gas mass fractions. Gas-rich galaxies thus had, on average, larger distances and therefore lower spatial resolution, making their bars harder to identify.

To see if the specific $f_{\text {bar }}$ trends of typical SDSS-based studies could be explained by these effects, I simulated SDSS observations by constructing mock samples using sampling with replacement from $\mathrm{S}^{4} \mathrm{G}$ galaxies with $D<30 \mathrm{Mpc}$, placing the galaxies at random (volume-weighted) redshifts between 0.01 and 0.05 ; $f_{\text {bar }}$ was then computed by assuming that bars with observed sizes less than twice the typical SDSS FWHM were not detected. This did an excellent job of reproducing the reported $f_{\mathrm{bar}}-\log \left(M_{\star} / M_{\odot}\right)$ trend from GZ2 studies, and at least partly reproduced the reported $f_{\text {bar }}-\log f_{\text {gas }}$ trends of Masters et al. (2012) and Cervantes Sodi (2017).

The absence of a clear trend between bar presence and gas content in local galaxies casts doubt on models in which bar formation is suppressed by high gas fractions in the disc, 
unless any such suppression is temporary only, and ended significantly before the present epoch.

Finally, I note that differential detection effects due to bar-size correlations and changing distances could also affect high-redshift studies of the bar fraction, since even HST's resolution is not good enough to prevent smaller bars from being missed, especially at higher redshifts. The apparent decrease in $f_{\text {bar }}$ reported for high-redshift samples - and the reported trends of $f_{\text {bar }}$ increasing to higher stellar masses over the range $\log \left(M_{\star} / M_{\odot}\right) \sim 10-11$ at high redshifts could also be influenced by secular changes in bar size: since most theoretical studies argue that bars grow in length over time, bars at higher redshifts would be smaller in size, and thus even harder to detect. Simulations similar to those done for the SDSS-based studies suggest that the combination of changing resolution and growth in bar sizes could account for at least some - though probably not all - of the reported evolution of bar fractions with redshift.

\section{ACKNOWLEDGMENTS}

This study benefited greatly from conversations with, and encouragement from, Victor Debattista. I also thank Dave Wilman and Ariel Sanchez for useful discussions and Martin Herrera-Endoqui for answering questions about the $\mathrm{S}^{4} \mathrm{G}$ measurements and comments on an earlier draft. Additional comments from Simón Díaz-García, Dimitri Gadotti, Karen Masters, Brooke Simmons, Sandor Kruk, Chris Lintott, and David Trinkle are greatly appreciated. I also thank the referee, Johan Knapen, for a number of helpful suggestions which improved this manuscript.

Funding for the creation and distribution of the SDSS Archive has been provided by the Alfred P. Sloan Foundation, the Participating Institutions, the National Aeronautics and Space Administration, the National Science Foundation, the U.S. Department of Energy, the Japanese Monbukagakusho, and the Max Planck Society. The SDSS Web site is http://www.sdss.org/.

The SDSS is managed by the Astrophysical Research Consortium (ARC) for the Participating Institutions. The Participating Institutions are The University of Chicago, Fermilab, the Institute for Advanced Study, the Japan Participation Group, The Johns Hopkins University, the Korean Scientist Group, Los Alamos National Laboratory, the MaxPlanck-Institute for Astronomy (MPIA), the Max-PlanckInstitute for Astrophysics (MPA), New Mexico State University, University of Pittsburgh, University of Portsmouth, Princeton University, the United States Naval Observatory, and the University of Washington.

This research also made use of Astropy, a communitydeveloped core Python package for Astronomy (Astropy Collaboration et al. 2013).

\section{REFERENCES}

Abraham R. G., Merrifield M. R., Ellis R. S., Tanvir N. R., Brinchmann J., 1999, MNRAS, 308, 569

Aguerri J. A. L., Méndez-Abreu J., Corsini E. M., 2009, A\&A, 495,491

Astropy Collaboration et al., 2013, A\&A, 558, A33

Athanassoula E., 2002, ApJL, 569, L83
Athanassoula E., 2003, MNRAS, 341, 1179

Athanassoula E., 2008, MNRAS, 390, L69

Athanassoula E., Machado R. E. G., Rodionov S. A., 2013, MNRAS, 429, 1949

Baillard A., et al., 2011, A\&A, 532, A74

Barazza F. D., Jogee S., Marinova I., 2008, ApJ, 675, 1194

Bennett C. L., et al., 2013, ApJS, 208, 20

Berentzen I., Heller C. H., Shlosman I., Fricke K. J., 1998, MNRAS, 300, 49

Bournaud F., Combes F., 2002, A\&A, 392, 83

Bournaud F., Combes F., Semelin B., 2005, MNRAS, 364, L18

Buta R., Laurikainen E., Salo H., Knapen J. H., 2010, ApJ, 721, 259

Buta R. J., et al., 2015, ApJS, 217, 32

Cameron E., et al., 2010, MNRAS, 409, 346

Cappellari M., et al., 2013, MNRAS, 432, 1862

Cervantes Sodi B., 2017, ApJ, 835, 80

Cheung E., et al., 2013, ApJ, 779, 162

Cheung E., et al., 2015, MNRAS, 447, 506

Consolandi G., 2016, A\&A, 595, A67

Consolandi G., Gavazzi G., Fumagalli M., Dotti M., Fossati M., 2016, A\&A, 591, A38

Cook D. O., et al., 2014, MNRAS, 445, 890

Debattista V. P., Sellwood J. A., 2000, ApJ, 543, 704

Debattista V. P., Mayer L., Carollo C. M., Moore B., Wadsley J., Quinn T., 2006, ApJ, 645, 209

Díaz-García S., Salo H., Laurikainen E., Herrera-Endoqui M., 2016a, A\&A, 587, A160

Díaz-García S., Salo H., Laurikainen E., 2016b, A\&A, 596, A84

Efstathiou G., Lake G., Negroponte J., 1982, MNRAS, 199, 1069

Elmegreen B. G., Elmegreen D. M., Hirst A. C., 2004, ApJ, 612, 191

Eskridge P. B., et al., 2000, AJ, 119, 536

Gadotti D. A., 2011, MNRAS, 415, 3308

Gavazzi G., et al., 2015, A\&A, 580, A116

Giovanelli R., Haynes M. P., 1988, Extragalactic neutral hydrogen. Springer-Verlag, pp 522-562

Giovanelli R., et al., 2005, AJ, 130, 2598

Goulding A. D., et al., 2017, ApJ, 843, 135

Haynes M. P., et al., 2011, AJ, 142, 170

Herrera-Endoqui M., Díaz-García S., Laurikainen E., Salo H., 2015, A\&A, 582, A86

Ho L. C., Filippenko A. V., Sargent W. L. W., 1997, ApJ, 487, 591

Hohl F., 1976, AJ, 81, 30

Holley-Bockelmann K., Weinberg M., Katz N., 2005, MNRAS, 363,991

Hoyle B., et al., 2011, MNRAS, 415, 3627

Jogee S., et al., 2004, ApJ, 615, L105

Knapen J. H., Shlosman I., Peletier R. F., 2000, ApJ, 529, 93

Knapen J. H., Erroz-Ferrer S., Roa J., Bakos J., Cisternas M., Leaman R., Szymanek N., 2014, A\&A, 569, A91

Kormendy J., Kennicutt Jr. R. C., 2004, ARA\&A, 42, 603

Laine S., Shlosman I., Knapen J. H., Peletier R. F., 2002, ApJ, 567, 97

Lee G.-H., Park C., Lee M. G., Choi Y.-Y., 2012a, ApJ, 745, 125

Lee G.-H., Woo J.-H., Lee M. G., Hwang H. S., Lee J. C., Sohn J., Lee J. H., 2012b, ApJ, 750, 141

Lintott C. J., et al., 2008, MNRAS, 389, 1179

Martinez-Valpuesta I., Shlosman I., Heller C., 2006, ApJ, 637, 214

Masters K. L., et al., 2011, MNRAS, 411, 2026

Masters K. L., et al., 2012, MNRAS, 424, 2180

Melvin T., et al., 2014, MNRAS, 438, 2882

Méndez-Abreu J., Sánchez-Janssen R., Aguerri J. A. L., Corsini E. M., Zarattini S., 2012, ApJ, 761, L6

Menéndez-Delmestre K., Sheth K., Schinnerer E., Jarrett T. H., Scoville N. Z., 2007, ApJ, 657, 790 
Mo H. J., Mao S., White S. D. M., 1998, MNRAS, 295, 319

Muñoz-Mateos J. C., et al., 2015, ApJS, 219, 3

Mulchaey J. S., Regan M. W., 1997, ApJ, 482, L135

Nair P. B., Abraham R. G., 2010a, ApJS, 186, 427

Nair P. B., Abraham R. G., 2010b, ApJ, 714, L260

Oh S., Oh K., Yi S. K., 2012, ApJS, 198, 4

Ostriker J. P., Peebles P. J. E., 1973, ApJ, 186, 467

Saha K., Pfenniger D., Taam R. E., 2013, ApJ, 764, 123

Sakov A., Golani I., Lipkind D., Benjamini Y., 2010, Ann. Applied Statistics, 4, 743

Salo H., et al., 2015, ApJS, 219, 4

Sellwood J. A., 2014, Reviews of Modern Physics, 86, 1

Sellwood J. A., 2016, ApJ, 819, 92

Sellwood J. A., Wilkinson A., 1993, Reports on Progress in Physics, 56, 173

Sheth K., Regan M. W., Scoville N. Z., Strubbe L. E., 2003, ApJ, 592, L13

Sheth K., et al., 2008, ApJ, 675, 1141

Sheth K., et al., 2010, PASP, 122, 1397

Sheth K., Melbourne J., Elmegreen D. M., Elmegreen B. G., Athanassoula E., Abraham R. G., Weiner B. J., 2012, ApJ, 758,136

Sheth K., et al., 2013, Not Dead Yet! Completing Spitzer's Legacy with Early Type Galaxies, Spitzer Proposal

Shlosman I., Frank J., Begelman M. C., 1989, Nature, 338, 45

Shlosman I., Begelman M. C., Frank J., 1990, Nature, 345, 679

Simmons B. D., et al., 2014, MNRAS, 445, 3466

Skibba R. A., et al., 2012, MNRAS, 423, 1485

Wang J., et al., 2012, MNRAS, 423, 3486

Weinberg M. D., Katz N., 2002, ApJ, 580, 627

Willett K. W., et al., 2013, MNRAS, 435, 2835

Wilman D. J., Erwin P., 2012, ApJ, 746, 160

Wilson E., 1927, J. Amerc. Statist. Assoc., 22, 209

York D. G., et al., 2000, AJ, 120, 1579

Yoshino A., Yamauchi C., 2015, MNRAS, 446, 3749

Zibetti S., Charlot S., Rix H., 2009, MNRAS, 400, 1181

\section{APPENDIX A: GALAXY COLOURS}

\section{A1 Correcting for Incompleteness in Galaxy Colours}

The HyperLeda $(B-V)_{\text {tc }}$ colours are the largest set of homogeneous, whole-galaxy colours available for very nearby galaxies such as those in the $\mathrm{S}^{4} \mathrm{G}$ sample. (SDSS colours are available for many more galaxies in general, but the sky coverage is limited and very nearby galaxies are prone to suffer from the "shredding" effect, so that they do not have accurate SDSS magnitudes or colours - see, e.g., Consolandi et al. 2016). Even so, the HyperLeda colours are highly incomplete: about half of the galaxies in the $\mathrm{S}^{4} \mathrm{G}$ sample do not have such colours. What is potentially worse is that the incompleteness is not random; as Figure A1 shows, it is a strong function of apparent magnitude.

To correct for this incompleteness, I weight individual galaxies in colour-based analyses by the inverse of their apparent-magnitude-based colour completeness. To generate these weights, I model the observed completeness of $(B-V)_{\text {tc }}$ values as a function of $B_{\mathrm{tc}}$ using a cubic Akima spline interpolation, ${ }^{10}$ as provided in the Scipy interpolate module

10 Akima splines provide a smoother interpolation, with less ringing between data points, than do a standard cubic splines.

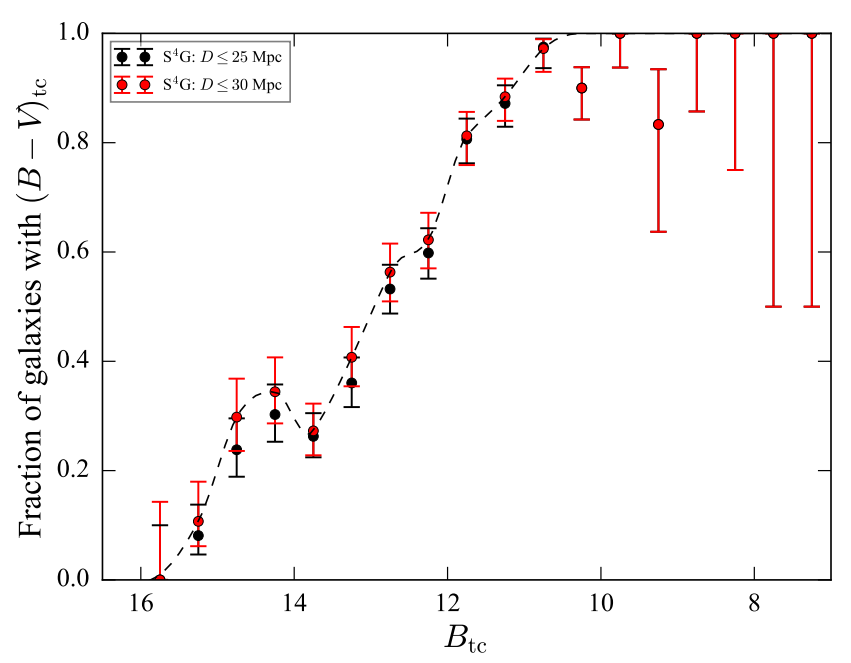

Figure A1. Completeness of $(B-V)_{\text {tc }}$ colours from HyperLeda for the $\mathrm{S}^{4} \mathrm{G}$ sample as a function of apparent blue magnitude $B_{\text {tc }}$ for the two distance-limited subsamples (black, red). The dashed black line shows the spline interpolation (to the $D \leqslant 30$ Mpc subsample) used to generate galaxy weights for colour-based analyses.

(curve in Figure A1). To allow the interpolation to asymptote smoothly to zero for faint $B_{\mathrm{tc}}$ values (and thus avoid potentially infinite weights for very faint galaxies), I added two artificial points at $B_{\mathrm{tc}}=16$ and 17 . Since only $\sim 1 \%$ of the sample galaxies are fainter than $B_{\mathrm{tc}}=15.5$, the effect is minimal.

In Section 3.2, I compute the $\mathrm{S}^{4} \mathrm{G}$ bar fraction as a function of $g-r$ colour, since the latter makes for a direct comparison with previously published SDSS bar studies (e.g., Barazza et al. 2008; Masters et al. 2011, 2012). The $g-r$ colours are based on the $(B-V)_{\mathrm{tc}}$ colours, using the empirical, galaxy-based transformations of Cook et al. (2014), which work out (from their Table 3) as $g-r=$ $1.12(B-V)-0.18$.

\section{A2 Bar Fractions as Function of $B-V$}

To test whether the colour transformation from $B-V$ to $g-r$ introduces any significant biases, I plot the bar fraction as a function of $(B-V)_{\mathrm{tc}}$ in Figure A2. Comparison with the bar fraction as a function of $g-r$ (middle panels of Figure 5 ) shows that the basic relation is unchanged: bar fraction is approximately constant across a broad range of colour, with some weak evidence for a decrease in the bar fraction for redder galaxies.

\section{APPENDIX B: COMPARISON OF RESULTS FOR ALTERNATE SUBSAMPLES}

\section{B1 Subsample 2 versus Subsample 1}

Most of the analysis in this paper uses $\mathrm{S}^{4} \mathrm{G}$ galaxies from Sample $1(D \leqslant 25 \mathrm{Mpc})$ or the mass-limited Sample $1 \mathrm{~m}$ $\left(D \leqslant 25 \mathrm{Mpc}\right.$ and $\left.\log \left(M_{\star} / M_{\odot}\right) \geqslant 8.5\right)$. In this section, 

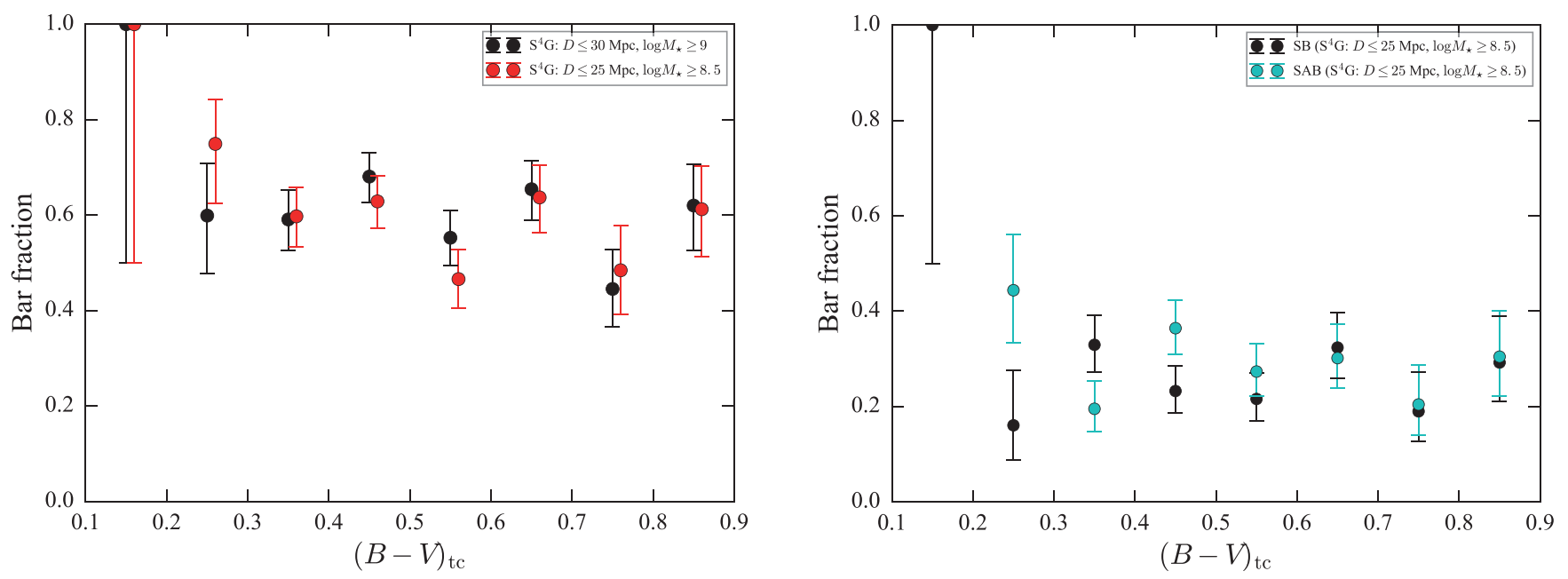

Figure A2. Left: Fraction of disc galaxies with bars as a function of total, extinction-corrected $B-V$ colour for $S^{4} \mathrm{G}$ galaxies in Samples $1 \mathrm{~m}$ (black) and $2 \mathrm{~m}$ (red). Right: Fractions of disc galaxies with strong (black) and weak (cyan) bars as a function of total, extinction-corrected $B-V$ colour for Sample $2 \mathrm{~m}\left(D \leqslant 30 \mathrm{Mpc}, \log \left(M_{\star} / M_{\odot}\right) \geqslant 9\right)$. These should be compared with the plots of bar fraction versus $g-r$ colour in the middle panels of Figure 5 .

I compare the effects of using Samples $2(D \leqslant 30 \mathrm{Mpc})$ and $2 \mathrm{~m}\left(D \leqslant 30 \mathrm{Mpc}\right.$ and $\left.\log \left(M_{\star} / M_{\odot}\right) \geqslant 9\right)$. Figure B1 is equivalent to Figure 5, except that bar frequencies using both samples are plotted against $\log \left(M_{\star} / M_{\odot}\right), g-r$, and $f_{\text {gas }}$; frequencies based on Samples 2 or $2 \mathrm{~m}$ are plotted using hollow circles. The overall trends are the same in all cases, with significant differences between the samples only in the bluest or reddest $g-r$ bins, where the number of galaxies is very small. For comparison, the bar fractions reported by Díaz-García et al. (2016a) based on their complete sample - the basis for the Parent Disc Sample of this paper - are plotted using gray squares.

\section{B2 Bar Fraction in S0 Galaxies}

Figure B2 shows the effects of excluding or including S0 galaxies in the sample, as well as the bar frequency (as a function of stellar mass) for just the S0 galaxies. The red points (left-hand panel) are the standard Sample $1 f_{\text {bar }}$ trend for spiral galaxies, identical to the trend in the upper left panel of Figure 5. Adding the S0 galaxies to Sample 1 produces a flatter trend for $\log \left(M_{\star} / M_{\odot}\right)>9.5$ (orange points). The right-hand panel compares the stellar-mass distributions of the spirals and the S0s in the Parent Disc Sample. In contrast to the spirals, whose mass distribution peaks at around $10^{9} M_{\odot}$, the S0 distribution peaks at $\sim 10^{10} M_{\odot}$; this helps explain why including the S0s mainly affects the higher-mass side of the $f_{\text {bar }}$ trend in the left-hand panel.

This paper has been typeset from a $\mathrm{T}_{\mathrm{E}} \mathrm{X} / \mathrm{LAT}_{\mathrm{E}} \mathrm{X}$ file prepared by the author. 

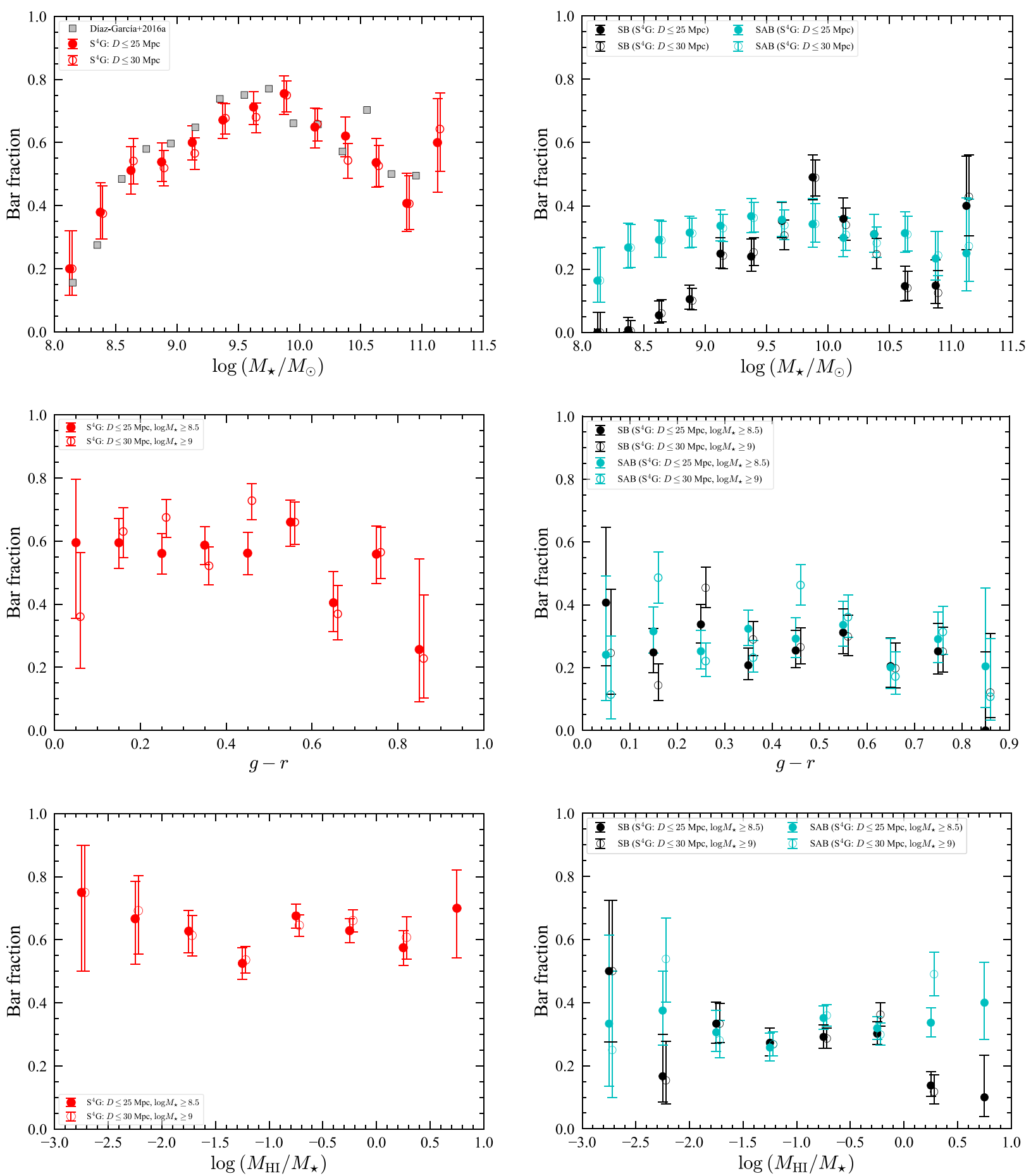

Figure B1. As for Figure 5, with filled circles showing bar frequencies for Samples 1 (top row) or $1 \mathrm{~m}$ (middle and bottom rows), but also showing bar frequencies for Samples 2 or $2 \mathrm{~m}$ using hollow circles. Points for Samples 2 and $2 \mathrm{~m}$ are slightly offset along the horizontal axes to make comparisons easer. For clarity, bar frequencies from other studies are not shown (see Figure 5); however, the upper-left panel does include the published bar frequencies for the parent sample as they appear in Fig. 19 of Díaz-García et al. (2016a). Note that Sample $2 \mathrm{~m}$ does not have enough galaxies in the highest- $f_{\text {gas }}$ bins for bar fractions to be calculated there (bottom panels). 

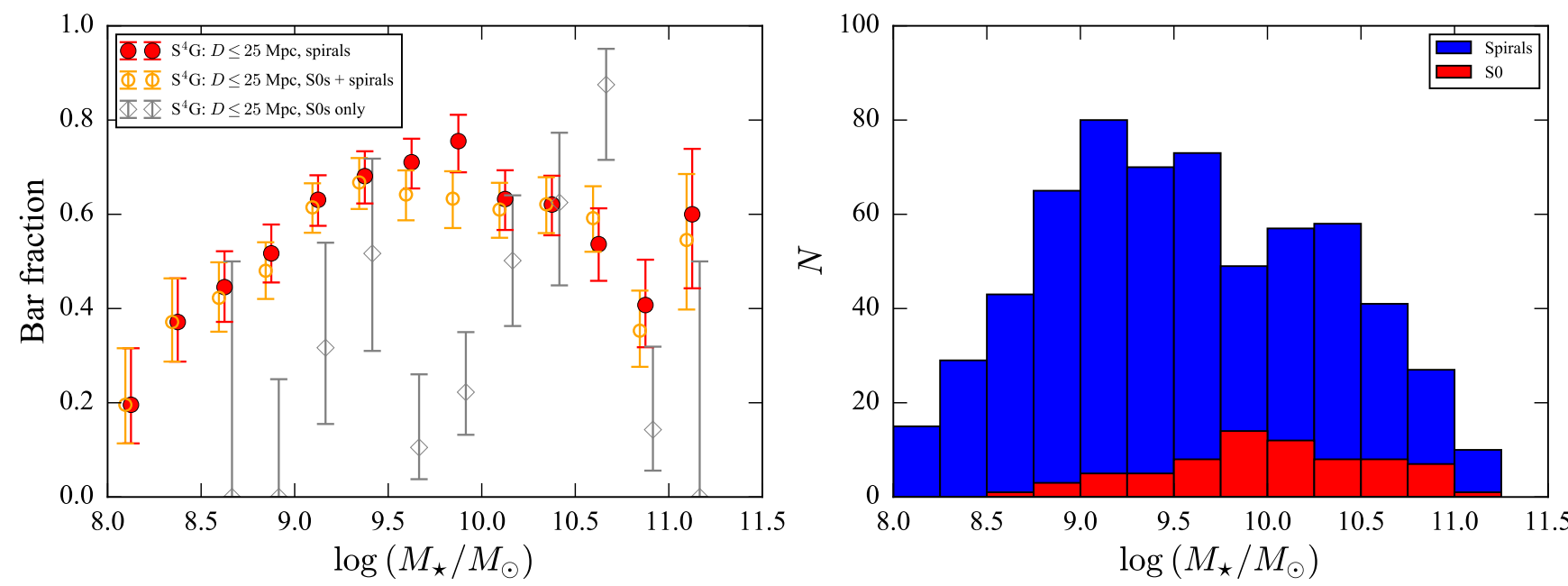

Figure B2. Left: Bar fraction as a function of stellar mass for Sample 1 spirals (red circles, same as in Figure 5), spirals + S0 galaxies with same distance limit (open orange circles), and S0 galaxies alone (open grey diamonds). The flatter values of $f_{\mathrm{bar}}$ between $\log \left(M_{\star} / M_{\odot}\right) \sim 9.5$ and 10.5 for the spirals + S0s sample compared to the pure-spirals sample is due primarily to the very low $f_{\text {bar }}$ values for S0 galaxies between $\log \left(M_{\star} / M_{\odot}\right) \sim 9.5$ and 10 and secondarily to the spike in S0 $f_{\mathrm{bar}}$ for $\log \left(M_{\star} / M_{\odot}\right) \sim 10.5$. Right: Distribution of stellar masses for spirals in Sample 1 (blue) and for S0s meeting same criteria (red). 